\title{
Independent Guidance of Retinal Axons in the Developing Visual System of Drosophila
}

\author{
Samuel Kunes, Clive Wilson, and Hermann Steller \\ Howard Hughes Medical Institute, Departments of Brain and Cognitive Sciences, and Biology, Massachusetts Institute of \\ Technology, Cambridge, Massachusetts 02139
}

The development of the adult visual system of Drosophila requires the establishment of precise retinotopic connections between retinal photoreceptor cell axons and their synaptic partners in the optic lobe of the brain. To assess the role of axon-axon interactions in retinal axon guidance, we used genetic methods to disrupt the normal spatiotemporal order of retinal axon ingrowth. We examined retinal axon projections to the developing first optic ganglion, the lamina, in two mutants in which reduced numbers of ommatidia develop in the eye imaginal disk. We find that in the developing lamina of these mutants, sine oculis and Ellipse, retinal axons project to proper dorsoventral positions despite the absence of the usual array of neighboring retinal axons. In a second approach, we examined animals that were somatic mosaics for the mutation, glass. In glass ${ }^{-}$animals, retinal axons project aberrantly and the larval optic nerve is absent. We find that in the developing lamina of glass mosaic animals, wild-type retinal axons project to proper dorsoventral positions despite the misrouted projections of neighboring

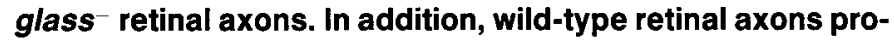
ject normally in the absence of the larval optic nerve, indicating that the latter is not an essential pioneer for retinal axon navigation. Our observations support the proposal that axon fascicles can make at least some pathfinding decisions independently of other retinal axon fascicles. We suggest that positional guidance cues that might label axon pathways and target destinations contribute to retinotopic pattern formation in the Drosophila visual system.

[Key words: retinal axon guidance, visual system development, retinotopic pattern formation, photoreceptor axons, optic lobe development, Drosophila]

Received June 9, 1992; revised Aug. 19, 1992; accepted Aug. 21, 1992.

We thank Dr. S. Benzer (CalTech) for providing the monoclonal antibodies (mAb 24B10 and mAb 22Cl0) used in this study and Dr. Kevin Moses (USC) and Dr. Gerry Rubin (UC Berkeley) for providing the $g^{600}$ stock and the transgenic strain harboring $\mathrm{P}\left[g l^{+}\left(10 \mathrm{~kb}\right.\right.$. Sal), $\left.r y^{+}\right]$. We thank Dr. Nicholas Baker (AECOM) for providing the $E l p^{\mathrm{B} 1}$ strain and for communicating results prior to publication. We thank our colleagues in the Steller laboratory for their comments on the manuscript and Dave Smith for assistance with confocal microscopy. Confocal microscopy was performed in a facility supported by the Lucille P. Markey Charitable Trust. S.K. was supported by a Damon-Runyon Walter Winchell Cancer Research Fund fellowship (DRG-994). C.W. was supported by a Charles A. King Trust fellowship from the Medical Foundation (Boston). This work was supported in part by a Pew Scholars Award to H.S. H.S. is an Assistant Investigator of the Howard Hughes Medical Institute.

Correspondence should be addressed to Dr. Hermann Steller, E25-436, Howard Hughes Medical Institute, Department of Brain and Cognitive Sciences, Massachusetts Institute of Technology, 45 Carlton Street, Cambridge, MA 02139.

Copyright (C) 1993 Society for Neuroscience $0270-6474 / 93 / 130752-16 \$ 05.00 / 0$
How the visual system achieves its precise and orderly pattern of interconnecting neurons has long been in question. Possible mechanisms include chemoaffinity (Sperry, 1963), which proposes that target neurons bear chemical labels selectively recognized by the appropriate retinal axons, and morphogenetic assembly (Gaze, 1960; Horder and Martin, 1978; Bodick and Levinthal, 1980; Grant and Rubin, 1980), in which the proper assembly of unlabeled neuronal components is specified by a defined spatial and temporal order of retinal axon outgrowth (reviewed in Cowan and Hunt, 1985; Udin and Fawcett, 1988). In the vertebrate visual system, there is strong support from in vitro experiments for a role of positional signaling molecules in retinal axon navigation (e.g., Bonhoeffer and Huf, 1982; Vielmetter and Stuermer, 1989; Stahl et al., 1990; see also Stirling, 1991). Additionally, a number of studies, particularly in invertebrates, suggest that a defined morphogenetic order of retinal axon outgrowth might play a central role in the development of retinotopic connectivity (e.g., Meinertzhagen, 1974; Anderson, 1978; Bunt et al., 1978; Macagno, 1978; Stuermer and Easter, 1984).

The Drosophila visual system offers a unique opportunity to examine the cellular and molecular events underlying axon guidance. The compound eye is organized into approximately 800 ommatidial units, each containing eight photoreceptor neurons (R-cells; reviewed in Strausfeld, 1976). The six outer photoreceptors, R1-R6, synapse to target neurons in the first optic ganglion, the lamina. The two central photoreceptors, R7 and $\mathrm{R} 8$, terminate in different layers of the medulla ganglion. Within these optic ganglia, photoreceptor axons connect to target neurons in a precise retinotopic array (Ramón y Cajal and Sánchez y Sánchez, 1915; Trujillo-Cenóz and Melamed, 1966; Braitenberg, 1967; Horridge and Meinertzhagen, 1970; Fischbach and Dittrich, 1989; reviewed in Strausfeld, 1976).

The development of this precise connection pattern begins in the third larval instar as photoreceptor neurons differentiate in the eye imaginal disk epithelium (Ready et al., 1976; Tomlinson and Ready, 1987; Tomlinson, 1988; Rubin, 1989) and send their axons into the developing optic ganglia of the brain (Fig. 1; Meinertzhagen, 1973; Trujillo-Cenóz and Melamed, 1973). Photoreceptor growth cones traverse long distances and make a number of stereotypic pathfinding decisions en route to their specific target destinations. The eight axons of a developing ommatidium bundle together into a fascicle that exits the posterior of the eye disk and traverses an epithelial tube (the optic stalk) alongside of the larval optic nerve (Meinertzhagen, 1973; Trujillo-Cenóz and Melamed, 1973), a component of the larval 
visual system (Bolwig, 1946). After exiting the optic stalk, retinal fascicles grow along the lateral surface of the brain until reaching specific positions, from which they dive toward their respective target destinations at the medial border of the developing lamina (Fig. $1 B$ ). Though the R1-R6 axons terminate here, the R7 and R8 axons continue their growth toward target destinations in the developing medulla. In both of these target areas, the arriving axons project to relative positions that correspond retinotopically to the positions of their origin in the eye disk epithelium.

We have sought to address the importance of two different possible mechanisms for retinal axon guidance in Drosophila. Retinal axons might be guided to their target destinations on the basis of the timing of axon outgrowth and interactions with other retinal axon fascicles. In Drosophila, as in a number of other organisms, a developmental role for selective interactions between axon fascicles is well documented (e.g., Goodman et al., 1984; Grenningloh et al., 1991). Given the strict spatiotemporal order of retinal axon outgrowth in the Drosophila visual system (Meinertzhagen, 1973, 1974; Trujillo-Cenóz and Melamed, 1973; Tomlinson and Ready, 1987; see Results for details), a growing fascicle could find its proper destination by following the fascicle from a nearby, more mature ommatidium. Because of the one-to-one order of the retinotopic map, this neighboring fascicle would project to a target position immediately adjacent to the proper destination for the new fascicle. An alternative mechanism for retinal axon guidance is that retinal axons make their pathfinding choices independently of one another, responding autonomously to guidance cues that label the appropriate paths and target sites.

This issue was long ago addressed in the retinotectal systems of lower vertebrates by approaches that included ablating various sections of the retina (Attardi and Sperry, 1963; Sperry, 1963; reviewed in Cowan and Hunt, 1985). Despite the absence of the normal array of neighboring retinal axons, remaining retinal ganglion cells are found to send their axons to roughly the correct target locales, leaving unoccupied the positions of the missing axons. These observations argued in favor of a mechanism involving chemoaffinity in the early formation of the retinotectal projection in these systems. However, analogous experiments performed in the small crustacean Daphnia (Macagno, 1978) and the locust Schistocerca (Anderson, 1978) suggest that the timing of axon outgrowth and axon-axon interactions may play a more central role in invertebrate visual systems (see Discussion for details).

To assess the importance of the spatiotemporal order of retinal axon outgrowth for retinal axon guidance in Drosophila, we examined two mutants, sine oculis (so; Milani, as referenced in Lindsley and Zimm, 1992) and Ellipse (Elp; Grell, as referenced in Lindsley and Zimm, 1992; Baker and Rubin, 1989), in which the number of ommatidia in the developing retina is reduced. In so and Elp larvae, we asked whether retinal axons project to appropriate lamina target positions despite the absence of the normal array of neighboring axons. In a second approach, we examined animals mosaic for the mutation glass $(g l)$. In larvae lacking the function of the $g l$ gene, retinal axons navigate aberrantly (Selleck and Steller, 1991) and the larval optic nerve is absent (Moses et al., 1989). In mosaic larvae, we determined whether wild-type retinal axons navigate normally, unlike their misrouted $\mathrm{gl}^{-}$neighbors. Our observations support the proposal that axon fascicles can make at least some pathfinding decisions independently of their neighbors. This suggests that positional guidance cues that label axon pathways and target
A

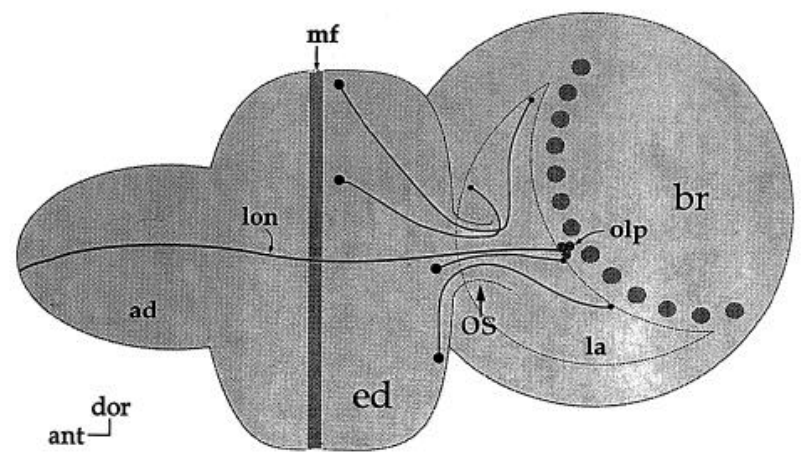

B

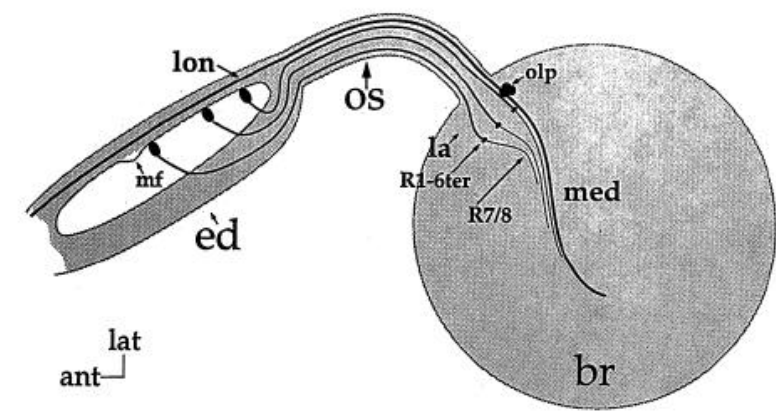

Figure 1. Structure of the developing adult visual system. A, Lateral view of the eye imaginal disk (ed), optic stalk (os), and brain $(b r)$ in the late third instar larval stage. The eye disk shows four ommatidia (solid circles) of the approximately 500 developing ommatidia present at this stage. The eight R-cells of each developing ommatidium send their axonal projections together in a fascicle (solid line) through the optic stalk alongside of the larval optic nerve (lon) into the developing lamina (the crescent-shaped region, $l a$ ). The larval optic nerve dives medially near a set of two or three cells, the OLPs (olp; Tix et al., 1989a), located on the dorsoventral midline at the posterior margin of the developing lamina. The two older developing ommatidia in ventral locations at the posterior of the eye disk send their projections to corresponding ventral positions at the posterior of the developing lamina. The two ommatidia in dorsal positions in the anterior of the eye disk (just posterior of the morphogenetic furrow; darkly shaded area labeled $\mathrm{mf}$ ) send their projections to corresponding dorsal positions at the anterior of the developing lamina. $a d$, antennal disk. $B$, Horizontal view of the structures shown in $A$. Three developing ommatidia distributed along the anteroposterior axis in the eye disk $(e d)$ are shown sending projections to corresponding positions in the developing lamina $(l a)$. The R1-R6 axons terminate $(R I-6 t e r)$ in the medial epithelial cell layer of the lamina, while the $\mathrm{R} 7$ and $\mathrm{R} 8$ axons $(R 7 / 8)$ project deeper to specific target sites in the developing medulla (med). In $A$, anterior (ant) is left, dorsal (dor) up. In $B$, anterior (ant) is left, lateral (lat) up.

destinations may contribute to retinotopic pattern formation in the Drosophila visual system.

\section{Materials and Methods}

Drosophila strains and culture. Strains harboring $\mathrm{P}\left[\mathrm{gl} \mathrm{l}^{+}\left(10 \mathrm{~kb}\right.\right.$. Sal), $\left.r \mathrm{y}^{+}\right]$ and the $g l^{60 J}$ mutation were obtained from K. Moses (USC) and G. M. Rubin (UC Berkeley). The $E l p^{\mathrm{B} 1}$ stock was obtained from N. Baker (AECOM). All fly strains were grown on standard cornmeal medium (Cline, 1978) at $18^{\circ} \mathrm{C}$ or $25^{\circ} \mathrm{C}$. Egg collections were performed as described by Ashburner (1989).

Generation of glass somatic mosaics. glass somatic mosaics were generated by inducing the somatic loss of a glass ${ }^{+}$P-element transgene 
(Moses et al., 1989) in a strain homozygous for a $g l$ null mutation, $g l^{60 \mathrm{~s}}$ (Moses et al., 1989). Females of the strain $\mathrm{P}\left[g l^{+}\left(10 \mathrm{~kb}\right.\right.$. Sal), $\left.r y^{+}\right] ; g 6^{60]}$ were crossed to $g l^{600}, \mathrm{P}\left[r y^{+}, \Delta 2-3\right] 99 \mathrm{~B}$ males. The $\mathrm{P}\left[r y^{+}, \Delta 2-3\right] 99 \mathrm{~B}$ insertion (Laski et al., 1986; Robertson et al., 1988) provides a constitutive source of $\mathbf{P}$ transposase in the soma of the offspring, which excises the glass $^{+}$element in some cells, resulting in mitotic clones of $\mathrm{gl}^{60 \mathrm{~J}}$ tissue.

Immunohistochemistry. For immunohistochemistry, the larval CNS and attached imaginal disk epithelia were dissected from climbing late third instar larvae and fixed in $4 \%$ paraformaldehyde ( $\mathrm{pH} 7.4$ ) overnight at $4^{\circ} \mathrm{C}$. After several washes in PBT (130 mM NaCl, 7 mм Na${ }_{2} \mathrm{HPO}_{4}$, $3 \mathrm{mM} \mathrm{NaH}_{2} \mathrm{PO}_{4}, 0.3 \%$ Triton X-100, $\mathrm{pH} 7.4$ ), the tissues were blocked for $30 \mathrm{~min}$ in BSTN [BSS (Ashburner, 1989) containing 10\% filtered goat serum (GIBCO), $0.3 \%$ Triton X-100]. Incubation with monoclonal antibody (mAb) 24B10 (Fujita et al., 1982; monoclonal supernatant diluted $1: 3$ in BSTN) overnight at $4^{\circ} \mathrm{C}$ with slow shaking was followed by four washes (20 min each) in PBT. After blocking with BSTN as before, the samples were incubated with secondary antibody [either RITC-conjugated goat anti-mouse IgG or HRP-conjugated goat antimouse IgG (Bio-Rad) diluted 1:100 in BSTN] overnight at $4^{\circ} \mathrm{C}$ and washed as before. For double labeling with $\mathrm{mAb} 24 \mathrm{~B} 10$ and anti-HRP (Jan and Jan, 1982), fluorescein isothiocyanate (FITC)-conjugated goat anti-HRP (Cappel) was included along with the rhodamine isothiocyanate (RITC)-conjugated goat anti-mouse (Cappel) secondary. Samples to be viewed by FITC or RITC epifluorescence were mounted in $70 \%$ glycerol, 0.1\% phenylenediamine in PBS (Johnson and Araujo, 1981). Samples reacted with the HRP-conjugated secondary antibody were stained using diaminobenzidine as described by Steller et al. (1987).

Axonal tracing with the carbocyanine dyes DiI and DiO. Following dissection in PBS, the CNS and attached imaginal disk epithelia were incubated for $30 \mathrm{~min}$ at $22^{\circ} \mathrm{C}$ in $150 \mathrm{U} / \mathrm{ml}$ collagenase type VII (Sigma) in PBS. This removes the peripodial membrane surrounding the eye disk and brain to permit access of $1,1^{\prime}$-dioctadecyl-3,3,3', $3^{\prime}$-tetramethylindocarbocyanine perchlorate (DiI) and 3,3'-dioctadecyloxacarbocyanine perchlorate ( $\mathrm{DiO}$ ) (Molecular Probes) crystals to neuronal membranes. The tissue was gently transferred to a tube containing $4 \%$ paraformaldehyde fixative $(\mathrm{pH} 7.4)$ and incubated overnight at $4^{\circ} \mathrm{C}$. After several washes in PBS, the tissue was stored in PBS at $4^{\circ} \mathrm{C}$ for several days. DiI and DiO crystals, ground finc between two glass slides, were applied with a pulled capillary glass needle to the basal surface of the eye disk submersed in PBS. The dye was allowed to diffuse along neuronal membranes for $24 \mathrm{hr}$ at room temperature in PBS (as described by Godement et al., 1987), after which the tissues were mounted in PBS for examination by confocal microscopy.

Lineage tracing with TRIT' $C^{\prime}$-dextran. Tetramethylrhodamine isothiocyanate (TRITC)-dextran [MW 10,000, lysine fixable (D-1817); Molecular Probes] was dissolved at $100 \mathrm{mg} / \mathrm{ml}$ in $5 \mathrm{~mm} \mathrm{KCl}, 0.1 \mathrm{~mm}$ phosphate buffer $\mathrm{pH}$ 6.8. Syncytial (1-3 hr) embryos were collected from $r y^{506}$ adults, dechorionated, and mounted for injection as previously described (Ashburner, 1989). The dextran label was distributed along the anteroposterior axis in the posterior two-thirds of the embryo. It quickly diffused throughout the embryo. Survival of injected embryos was unusually low (about 10\%), suggesting that the TRITC-dextran is somewhat toxic. Labeled animals were reared at $18^{\circ} \mathrm{C}$ in the dark until late third instar. After dissection, the larval CNS and attached imaginal tissues were fixed in $4 \%$ paraformaldehyde, stained with anti-HRP, and mounted for examination by confocal microscopy in $70 \%$ glycerol, $0.1 \%$ phenylenediamine (Johnson and Araujo, 1981) in PBS.

Confocal microscopy. Specimens were viewed under a Bio-Rad MRC600 confocal microscope equipped with a krypton/argon laser. Fluorescein and rhodamine fluorescence was detected using the BHS and YHS filter blocks, respectively. DiI fluorescence was detected with the YHS filter block while the BHS filter block detected both DiI and DiO. For each picture, approximately 30 images were collected, Kalmanaveraged, and when appropriate, subjected to edge-sharpening routines (using the Bio-Rad som software).

\section{Results \\ Retinotopic pattern of $R$-cell projections in the brain of a wild- type larva}

A progression of R-cell differentiation and ommatidial assembly sweeps posterior to anterior across the eye disk epithelium during the third larval instar and early pupal stages (Fig. 1; Ready et al., 1976; Tomlinson, 1988; Ready, 1989; Rubin, 1989). The anterior boundary of this progression is marked by the morphogenetic furrow, an indentation running from dorsal to ventral across the disk that delineates the onset of R-cell neuronal development and the formation of ommatidial cell clusters. Each dorsoventral row of developing ommatidia thus differs in age, with older rows toward the posterior of the disk. The number of ommatidia that develop in a dorsoventral row increases from several at the posterior margin to about 30 at the anteroposterior midline and decreases to several at the anterior margin of the disk.

R-cell axonogenesis begins immediately posterior of the morphogenetic furrow concomitant with R-cell neuronal differentiation (Tomlinson and Ready, 1987). Axon fascicles from a given dorsoventral row exit the eye disk, traverse the optic stalk, and enter the brain at about the same time. The first row of axon fascicles, derived from the posterior of the eye disk, occupies the most posterior row of target positions in the developing lamina (Fig. 1A,B; Meinertzhagen, 1973; Trujillo-Cenóz and Melamed, 1973). Each additional dorsoventral row of axon fascicles occupies the next available, more anterior row of target positions. Within each row, the dorsoventral order of retinal fascicles in the lamina target area appears to correspond in oneto-onc fashion with the relative order of thcir cell bodics in the eye disk.

In previous anatomical studies of the larval brain, the retinal axon projection pattern has been elucidated by the serial reconstruction of electron micrographs. A more convenient approach is to use antibody-staining and dye-tracing methods to label ommatidia and retinal axons differentially according to their developmental age and retinotopic position. We resolved these labeled axons' paths by confocal laser fluorescence microscopy. Photoreceptor cell bodies and axons, including those of the larval optic nerve, are specifically labeled by mAb $24 \mathrm{~B} 10$ (Fig. 2A; Fujita et al., 1982; Zipursky et al., 1984). Shown in Figure $2 B$ is a whole-mount stained with both $\mathrm{mAb} 24 \mathrm{~B} 10$ (red fluorescence) and anti-HRP (green fluorescence) and examined by confocal microscopy. Anti-HRP appears to label all neurons in Drosophila (Jan and Jan, 1982) including photoreceptor cells. Because the HRP antigens are expressed in differentiating R-cells approximately $9 \mathrm{hr}$ before the 24B10 antigen (also known as chaoptin; Zipursky et al., 1984; growth at $25^{\circ} \mathrm{C}$ ), the most anterior (youngest) six or seven rows of ommatidia are labeled by anti-HRP only. By using these antibodies together, it is possible to visualize the relative organization of young and old retinal axons in the optic stalk and brain.

Figure 2. Retinotopic organization of photoreceptor axons in the optic stalk and brain. All views, with the exception of $F$, are from the lateral perspective. $A$, Photoreceptor cells and their axons are shown labeled with mAb 24B10 (brown staining, Fujita et al., 1982) in a late third instar larval eye disk and brain. Retinal axons project through the optic stalk (os) alongside the larval optic nerve (lon) and terminate in retinotopic order in the developing lamina $(l a)$ and medulla (out of the focal plane). An arrowhead indicates the position of the morphogenetic furrow. $B$, As in $A$, but labeled with both mAb 24B10 (red fluorescence) and anti-HRP (green fluorescence) and viewed by confocal laser microscopy. Anti-HRP, which detects all neurons (Jan and Jan, 1982), stains R-cells and their axons about $9 \mathrm{hr}$ earlier in their development than mAb 24B10. The most anterior six or seven rows of ommatidia are therefore labeled by aiti-HRP only (not all anterior ommatidia are in the focial plane). The double-labeled (yellow) rows at the posterior of the eye disk send their axon fascicles to the posterior region of the developing lamina. An arrowhead indicates the 

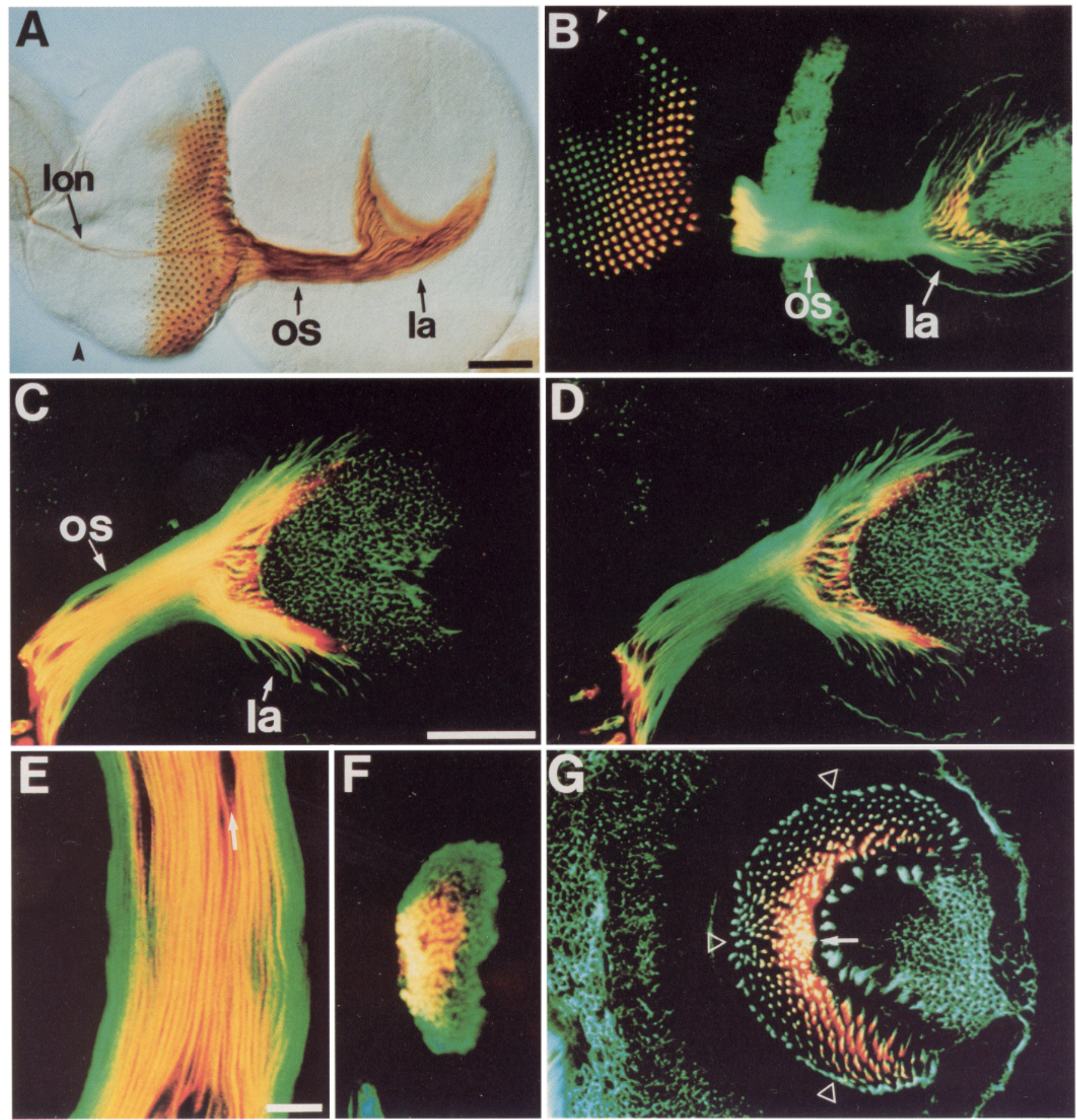

position of the morphogenetic furrow. $C$, Optical section through the lateral region of the optic stalk. Centrally located older (yellow) retinal fascicles are surrounded on either side by younger (green) fascicles labeled by Anti-HRP only. $D$, A slightly deeper focal plane than in $C$, showing only younger retinal fascicles in the medial region of the stalk and their tracts over the lateral surface of the brain to anterior, dorsal, and ventral positions within the developing lamina. $E$, A high-resolution view of the optic stalk in the focal plane shown in $C$. Individual fascicles, each containing the eight retinal axons of a single ommatidial unit, can be seen running in essentially parallel tracts through the stalk. Younger (green) fascicles occupy dorsal and ventral positions. Unstained gaps (like the one marked by the arrow) contain glial cells (data not shown). $F$, Transverse optical section through the optic stalk (an anterior view) showing retinal fascicles in transverse section. A fascicle appears as a single spot. Older (yellow) fascicles form a bundle on the lateral inner surface of the stalk membrane. Each new dorsoventral row of retinal fascicles adds concentrically at the periphery of the growing bundle. $G$, An optical section through the developing lamina, deeper than those shown in $C$ and $D$. Retinal fascicles are seen in transverse section as they dive toward the medial border of the lamina where R1-R6 terminate. The larval optic nerve is centrally located at the posterior margin adjacent to the OLPs, which are stained only by anti-HRP (position marked by arrow). Older (yellow) fascicles pass through the posterior of the developing lamina. Each successive (younger) dorsoventral row of retinal fascicles occupies the next available set of more anterior positions. The most anterior row is indicated by open arrowheads. In $A-D$ and $G$, anterior is at the left, dorsal at the top. In $E$ anterior is at the top, dorsal at the right. In $F$, lateral is at the left, dorsal at the top. Scale bars: $A, 25 \mu \mathrm{m}$ for $A$ and $B$; $C, 25 \mu \mathrm{m}$ for $C, D$, and $G ; E$, $5 \mu \mathrm{m}$ for $E$ and $F$. 

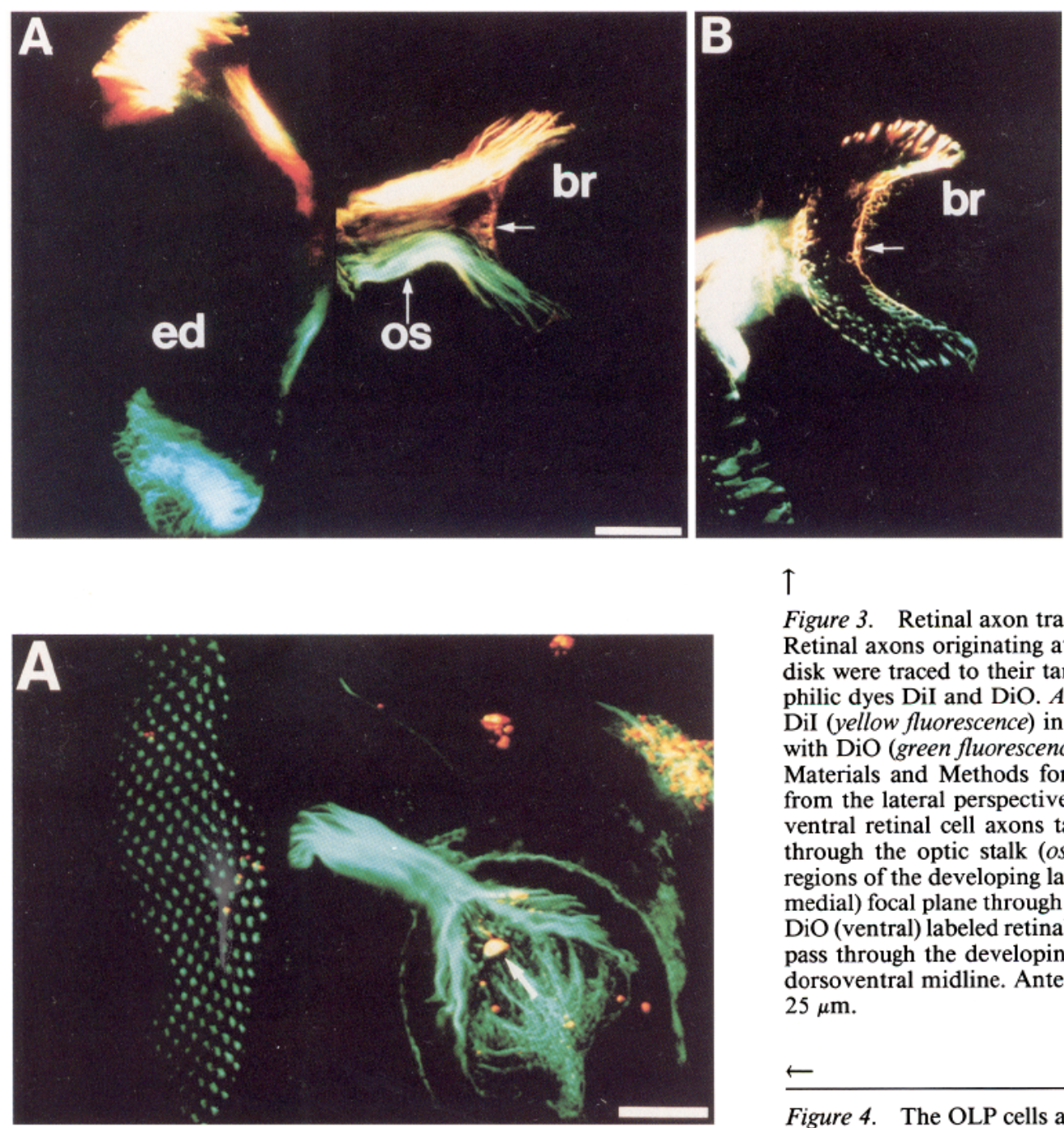

$\uparrow$

Figure 3. Retinal axon tracing using the lipophilic dyes DiI and DiO. Retinal axons originating at dorsal or ventral positions within the eye disk were traced to their targets in the brain by labeling with the lipophilic dyes DiI and DiO. $A$, Developing ommatidia were labeled with DiI (yellow fluorescence) in the dorsal region of the eye disk (ed) and with $\mathrm{DiO}$ (green fluorescence) in the ventral region of the eye disk (see Materials and Methods for details). Labeled specimens were viewed from the lateral perspective by confocal laser microscopy. Dorsal and ventral retinal cell axons take, respectively, dorsal and ventral tracts through the optic stalk $(o s)$ and enter respectively dorsal or ventral regions of the developing lamina in the brain $(b r)$. $B$, In a deeper (more medial) focal plane through the sample shown in $A$, the DiI (dorsal) and $\mathrm{DiO}$ (ventral) labeled retinal axons are seen in transverse section as they pass through the developing lamina. In $A$ and $B$, an arrow marks the dorsoventral midline. Anterior is toward the left, dorsal up. Scale bar, $25 \mu \mathrm{m}$.

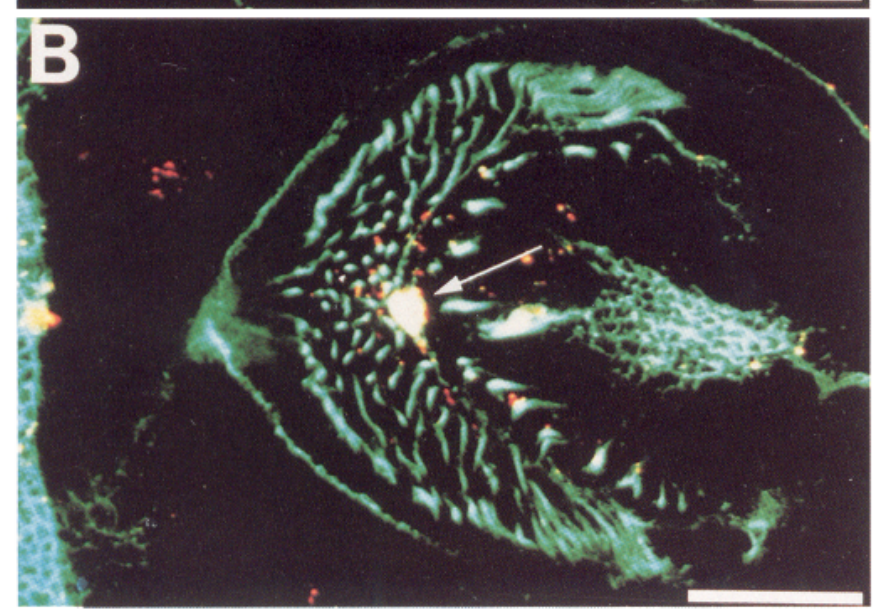

Figure 4. The OLP cells are a landmark for the dorsoventral midline of the developing lamina. To map retinal axon projections along the dorsoventral axis of the developing lamina, we used the OLP cells (Tix et al., 1989a) as a landmark for the dorsoventral midline. In samples stained with anti-HRP, we observed a group of two or three cells with characteristic punctate staining on the posterior margin of the developing lamina at the dorsoventral midline (see, e.g., Fig. $7 A, B$ ). Axons from these cells project medially, alongside of the larval optic nerve as it passes through the lamina toward the developing medulla. To confirm that these cells are indeed the OLP cells, we used a lineage tracer dye, TRITC-dextran, to label them specifically. The OLPs do not divide during the larval stages (Tix et al., 1989a) and thus retain the dextran tracer. $A$, A lateral view of the late third instar eye disk and brain after injection of TRITC-dextran (red) into the syncytial embryo and subsequent labeling with anti-HRP (green). The OLP cells are stained yellow by the combination of both labels (arrow). $B$, A deeper optical section through the developing lamina (lateral perspective) showing the position of the OLP cells (arrow) with respect to the retinal axon fascicles. In $A$ and $B$, anterior is toward the left, dorsal is up. In $A$, the brain is rotated slightly clockwise). Scale bars, $25 \mu \mathrm{m}$.

The retinal axon fascicles are resolved in considerable detail by confocal laser microscopy (Fig. $2 B-G$ ). In the optic stalk, the older retinal axons (stained yellow by the combination of both antibodies) form a cylindrical bundle apposing the inner lateral surface of the stalk membrane (Fig. $2 C-F$ ). Younger axon fascicles, labeled by anti-HRP only, surround the older fascicles on the dorsal, ventral, and medial sides. Retinal fascicles traverse the stalk well-ordered with respect to one another, rarely crossing over (Fig. 2E). In the brain, the older axon fascicles fan out in the posterior region of the developing lamina (Fig. $2 B, C$ ) while the younger fascicles occupy more anterior positions, or spread along the brain's lateral surface toward more dorsal and ventral positions (Fig. 2D). From these retinotopically specific positions, the fascicles dive medially through the lamina (Fig. 2G) toward the lateral border of the developing medulla. In the focal plane shown in Figure $2 G$, the fascicles 
within the developing lamina are viewed in transverse section, appearing as single spots. The oldest fascicles, which label most intensely with $\mathrm{mAb} 24 \mathrm{~B} 10$, are found in the central posterior region of the target area. Successively younger fascicle rows occupy more anterior positions. We have confirmed these observations by examining the relative organization of retinal axons using two other markers with temporally specific expression patterns: the antigen detected by mAb 22C10 (Fujita et al., 1982) and a $g l$ promoter-lacZ gene construct (construct C; Moses and Rubin, 1991; data not shown).

To examine the relative organization of $\mathrm{R}$-cell projections along the dorsoventral axis, we used the lipophilic dyes DiO and DiI (Sims et al., 1974, Honig and Hume, 1986; Godement et al., 1987) to trace retinal axons from specific positions in the eye disk to their target positions in the developing lamina (Fig. 3 ). In the specimen shown in Figure 3, R-cells from dorsal (DiIlabeled) and ventral (DiO-labeled) positions in the eye disk project their axons along, respectively, dorsal and ventral tracts in the optic stalk to corresponding dorsoventral positions in the developing lamina. A convenient landmark for the dorsoventral midline in the lamina target area is a group of two or three cells, the optic lobe pioneers (OLPs; Figs. $1 A, 2 G, 4 A, B$; Tix et al., 1989a), which are strongly labeled by anti-HRP (but not by $\mathrm{mAb} 24 \mathrm{~B} 10)$. Lineage tracing has shown that the OLP cells are born in embryos (Tix et al., 1989a; see also Fig. $4 A, B$ ). They associate with the larval optic nerve immediately posterior of the target field at the dorsoventral midline (Fig. 4B; Tix et al., 1989a).

These observations, consistent with the earlier works of Trujillo-Cenóz and Melamed (1973) and Meinertzhagen (1973, 1974), demonstrate that by the use of antibody-labeling and dye-tracing methods the early retinotopic order of the visual system can be resolved at the light microscopic level.

\section{Retinal axon projections in the mutant sine oculis}

In the eye disk of homozygous so larvae, ommatidia develop in reduced numbers or are entirely absent (Fig. 5). Ommatidia develop usually within a single area that can be found at different positions in different eye disks. Genetic mosaic analysis has shown the role of $s o$ in visual system development to be due to its function in the eye disk (Fischbach and Technau, 1984).

The position of a patch of developing ommatidia can be judged most reliably along the dorsoventral axis by using the larval optic nerve as a landmark for the dorsoventral midline in the eye disk (Fig. $5 A$ ). In the specimen shown in Figure $5 B$, a patch of developing ommatidia in the ventral region of the eye disk sends axons to the ventral region of the developing lamina. In the specimen shown in Figure $5 C$, a dorsal patch of ommatidia sends axons to the dorsal region of the lamina. Thus, our initial inspection of so animals suggested that retinal axons from these patches of developing ommatidia might project to appropriate regions of the developing lamina.

To resolve in greater detail the projections of retinal axons in $s o$ animals, we employed confocal microscopy. We examined the projections from patches of developing ommatidia with respect to the dorsoventral axis of the developing lamina, using the OLPs as a landmark for the midline in the brain. In so brains, the OLPs reflect the position of the midline reasonably well. This can be seen by comparing the OLPs' positions with those of the immediately posterior transmedullary fibers of the developing lobula complex (e.g., see Fig. 7B). Most so eye disks $(88 \%)$ did not contain developing ommatidia (Fig. 6; growth at
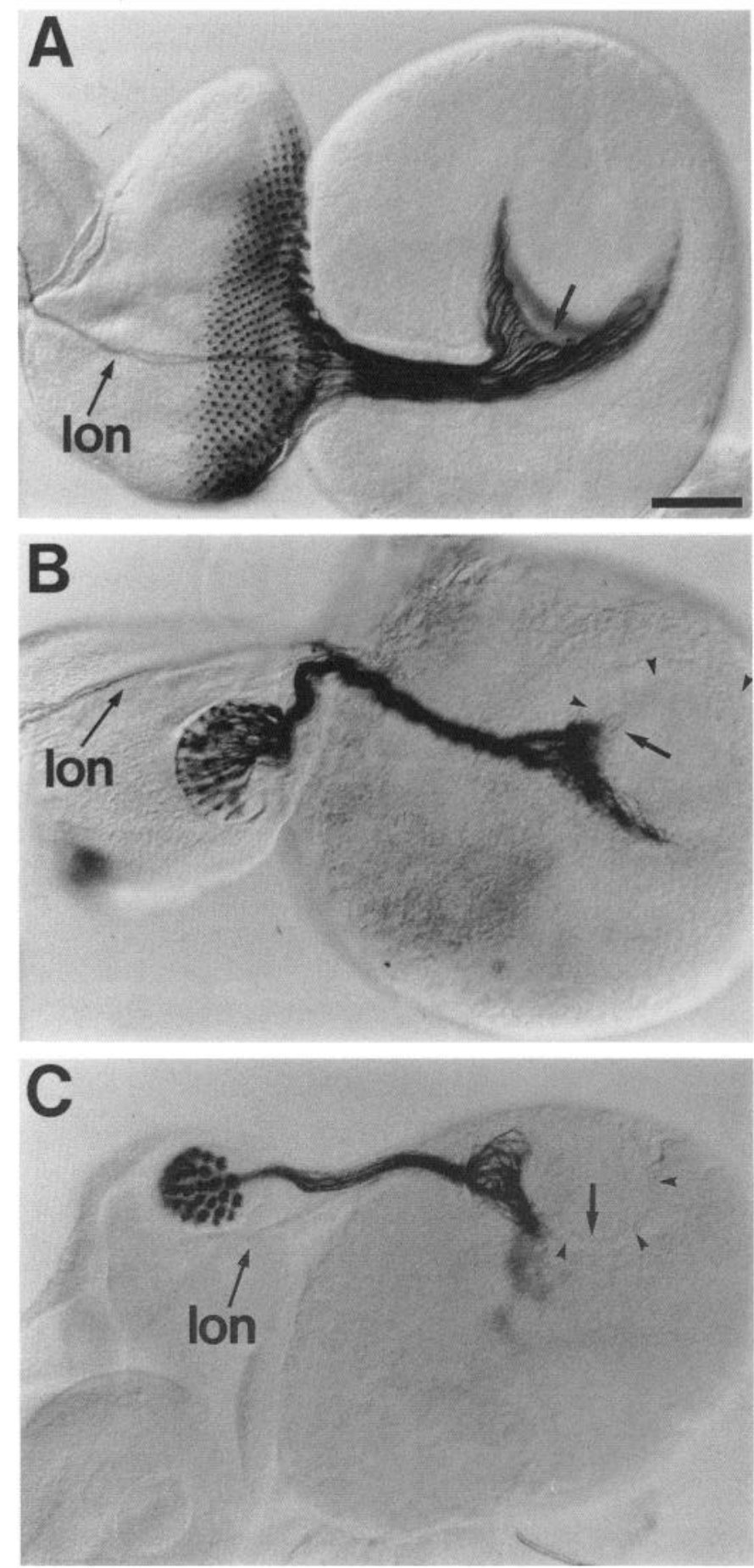

Figure 5. Retinal axon guidance in the mutant so. In so larvae, ommatidia develop in variously sized groups in different positions in the eye disk. Two so specimens with ventral $(B)$ and dorsal $(C)$ clusters of developing ommatidia are shown stained with $\mathrm{mAb} 24 \mathrm{~B} 10$ (dark staining). The larval optic nerve (lon) serves to mark the dorsoventral midline in the eye disk. In both specimens the retinal axons project appropriately to ventral $(B)$ and dorsal $(C)$ areas of the developing lamina (arrows mark the approximate location of the dorsoventral midline of the lamina), leaving the dorsal and ventral regions, respectively, unoccupied (regions marked by arrowheads). In $A$, wild-type is shown for comparison. Lateral perspectives are shown in $A-C$. Anterior is toward the left, dorsal is up. Scale bar, $25 \mu \mathrm{m}$. 


\section{Projection Pattern \#Observed}

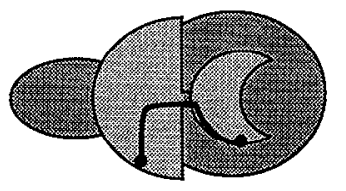

26

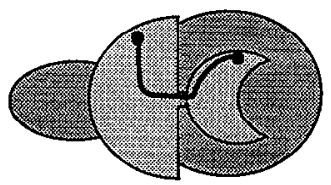

86

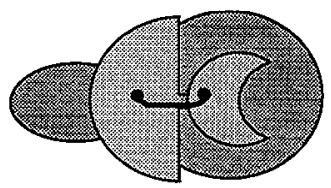

28

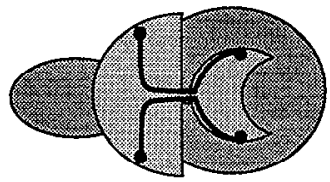

aberrant projection no photoreceptor cells imals resulted in similar observations for the fascicles from single isolated ommatidia (see below). However, we note that axons from these small patches of ommatidia often appeared to remain in multifascicle bundles within the lamina target area (see, e.g., Fig. 8C,F). Despite this aberrant behavior, the R1R6 axons in these bundles appeared to terminate within the developing lamina. In several specimens the eye disk harbored two discrete patches of developing ommatidia (Fig. $8 A-C$ ). In these cases, the retinal axons from different patches were observed to separate and project independently to the appropriate lamina regions. The analysis of retinal axon projections in so included a total of 148 specimens in which the eye disk harbored developing R-cells (Fig. 6). In all but four of these, retinal axons projected to appropriate dorsoventral positions. Thus, retinal axon fascicles must be capable of proper navigation in the absence of the full complement of neighboring axons.

\section{Projections of retinal axons in the mutant Ellipse}

Another mutation that reduces the number of developing ommatidia in the eye disk is $E l p$ (Grell, as referenced in Lindsley and Zimm, 1992; Baker and Rubin, 1989). Elp is a dominant gain-of-function allele of faint little ball ( $\mathrm{flb}$; Price et al., 1989; Schejter and Shilo, 1989), the gene encoding the Drosophila homolog of the epidermal growth factor receptor. $f l b^{+}$is an essential gene that acts at many stages of development; animals homozygous for the $f l b$ null mutation die as embryos. By contrast, dominant $E / p$ alleles appear to retain many of the normal functions of $f l b^{+}$. Homozygous $E l p$ animals are viable as adults. However, their eyes and wings develop abnormally, apparently due to increased levels of a normal $\mathrm{flb}^{+}$activity in the mutant (Baker and Rubin, 1989). The eyes of homozygous Elp flies contain only about $10 \%$ of the normal number of ommatidia. This defect is first detected in the eye imaginal disk of third instar larvae, where ommatidial clusters containing the normal complement of photoreceptor neurons are separated from each other by unusually large distances, particularly in the more anterior region of the disk (e.g., see Fig. $9 A, C, E$ ). Frequently, single anterior ommatidia or small groups of ommatidia are completely isolated from all other ommatidia. We were especially interested in examining the projection pattern of axons from these ommatidia.

An eye imaginal disk from a homozygous $E l p^{B l}$ animal is shown stained with anti-HRP and $\mathrm{mAb} 24 \mathrm{~B} 10$ antibodies in Figure $9 \mathrm{~A}$. The irregularly arranged ommatidia are most widely spaced in the morc anterior regions of the disk. These anterior ommatidia, like those in normal disks, stain only with antiHRP. Axons from the youngest ommatidia sometimes fail to enter the optic stalk at the posterior edge of the eye disk (data not shown). However, all other axons project to the larval brain through the optic stalk, although their paths to the stalk are frequently more circuitous than normal. As previously reported, the majority of photoreceptor clusters are concentrated at the posterior edge of the eye disk (Baker and Rubin, 1989; Zak and Shilo, 1992; N. Baker, personal communication). At least some of these ommatidia are thought to originate from more anterior, scattered positions and to assume more posterior locations as development proceeds.

The arrangement of fascicles in the lamina of $E l p^{B 1}$ larvae, though more disorganized, shares several features in common with wild-type larvae (see Fig. 9B). Fascicles first spread out over the lateral surface of the brain and then dive into the developing lamina; axons from the most mature ommatidia across a target rcgion unoccupied due to absence of the corresponding ommatidia. Our study of axon projections in Elp an- 

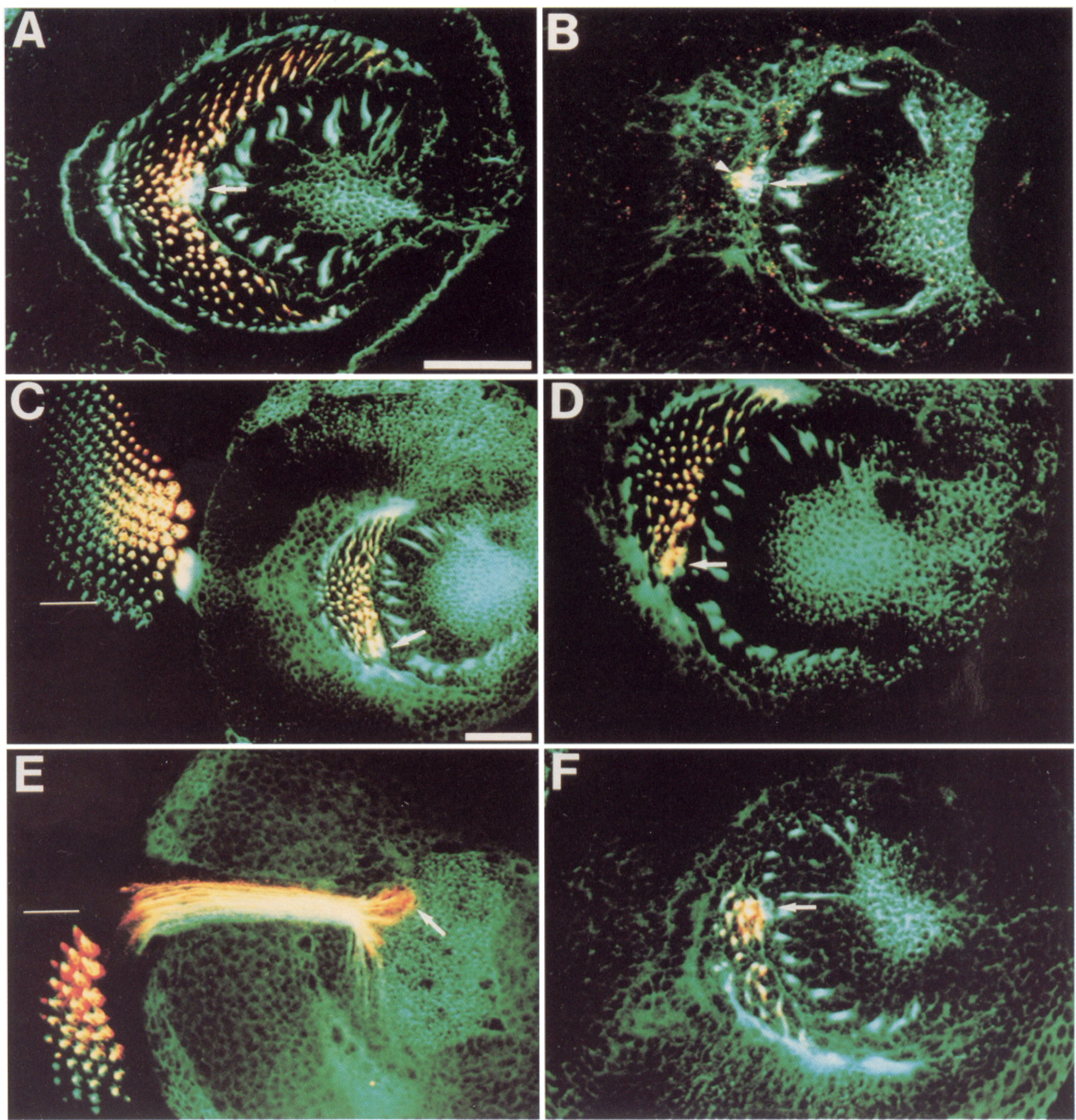

Figure 7. Confocal microscopic analysis of retinal axon projections in so larvae. All panels are from the lateral perspective. A, Optical section through the developing lamina of a wild-type larval brain stained with mAb 24B10 (red) and anti-HRP (green). A similar section is shown in Figure $2 G$. An arrow indicates the position of OLP cells, which label with anti-HRP only. $B$, Optical section like that shown in $A$ of an so brain completely lacking retinal innervation. The OLP cells and the larval optic nerve (a yellow spot in transverse section) are clearly visible posterior of the developing lamina at the dorsoventral midline (position indicated by arrow). On either side of the arrow, posterior of the lamina target area, the transmedullary fibers of the developing lobula complex are seen in approximate transverse section, stained by anti-HRP (green) and appearing as bright, elongated spots. These fibers are arrayed symmetrically on either side of the OLPs. $C$, Optical section including the eye disk and brain of an so specimen harboring about 200 developing ommatidia on the dorsal side of the midline (marked by a thin bar). $D$, Higher-magnification view of the developing lamina of the specimen shown in $C$. Retinal axon fascicles project to the dorsal side of the OLP cells (position indicated by arrow). E. An so specimen harboring about 40 developing ommatidia in the ventral region of the eye disk (midline marked by a thin bar). $F$, Optical section through the developing lamina of the specimen shown in $E$. Retinal axon fascicles project to ventral positions (arrow indicates the position of the OLPs). In all panels, anterior is toward the left, dorsal is up. Scale bars: $A, 25 \mu \mathrm{m}$ for $A, B, D$, and $F ; C, 25 \mu \mathrm{m}$ for $C$ and $E$. 

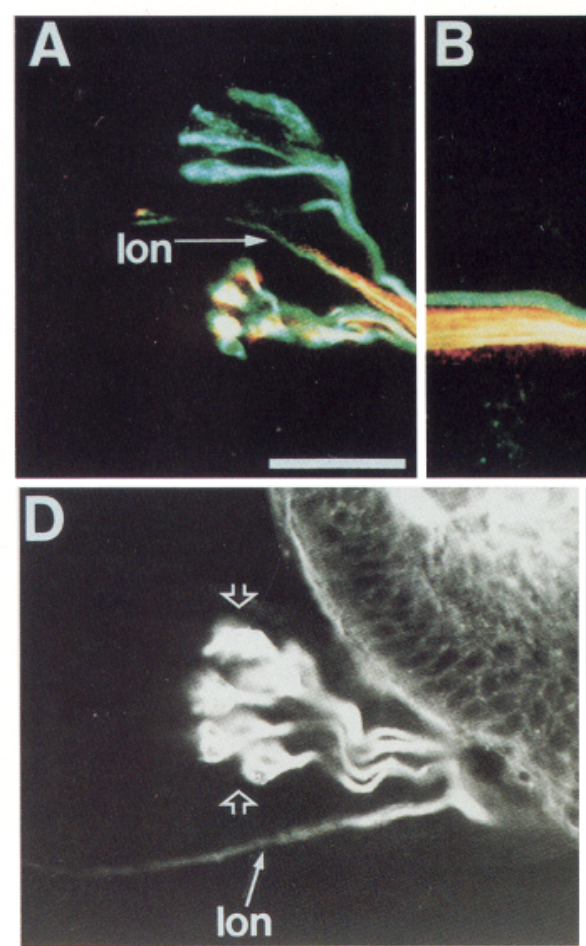

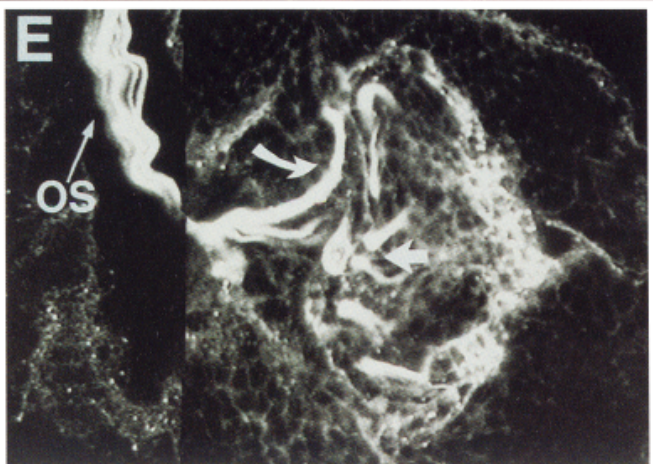

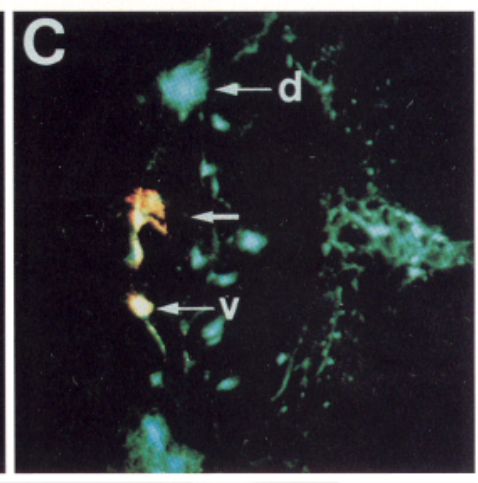

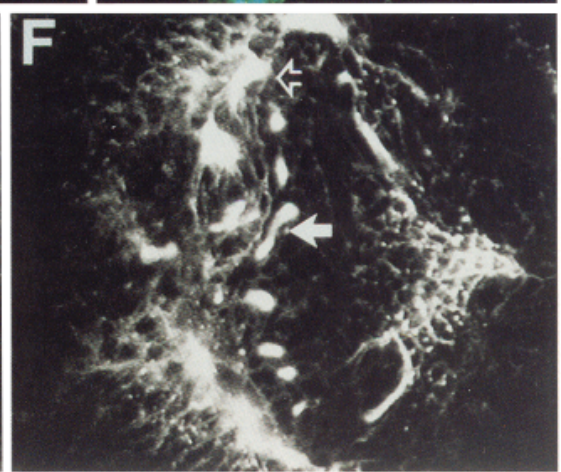

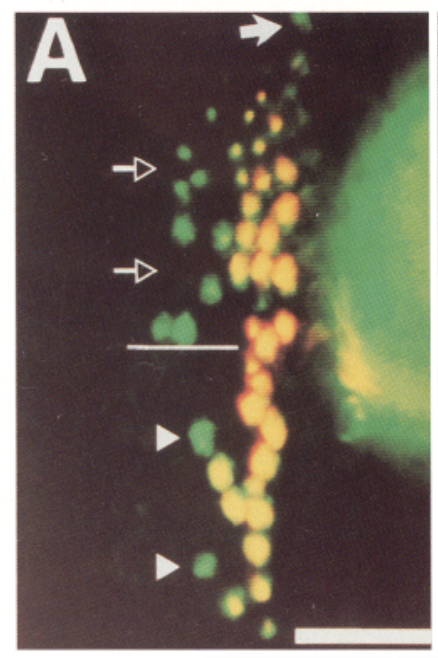
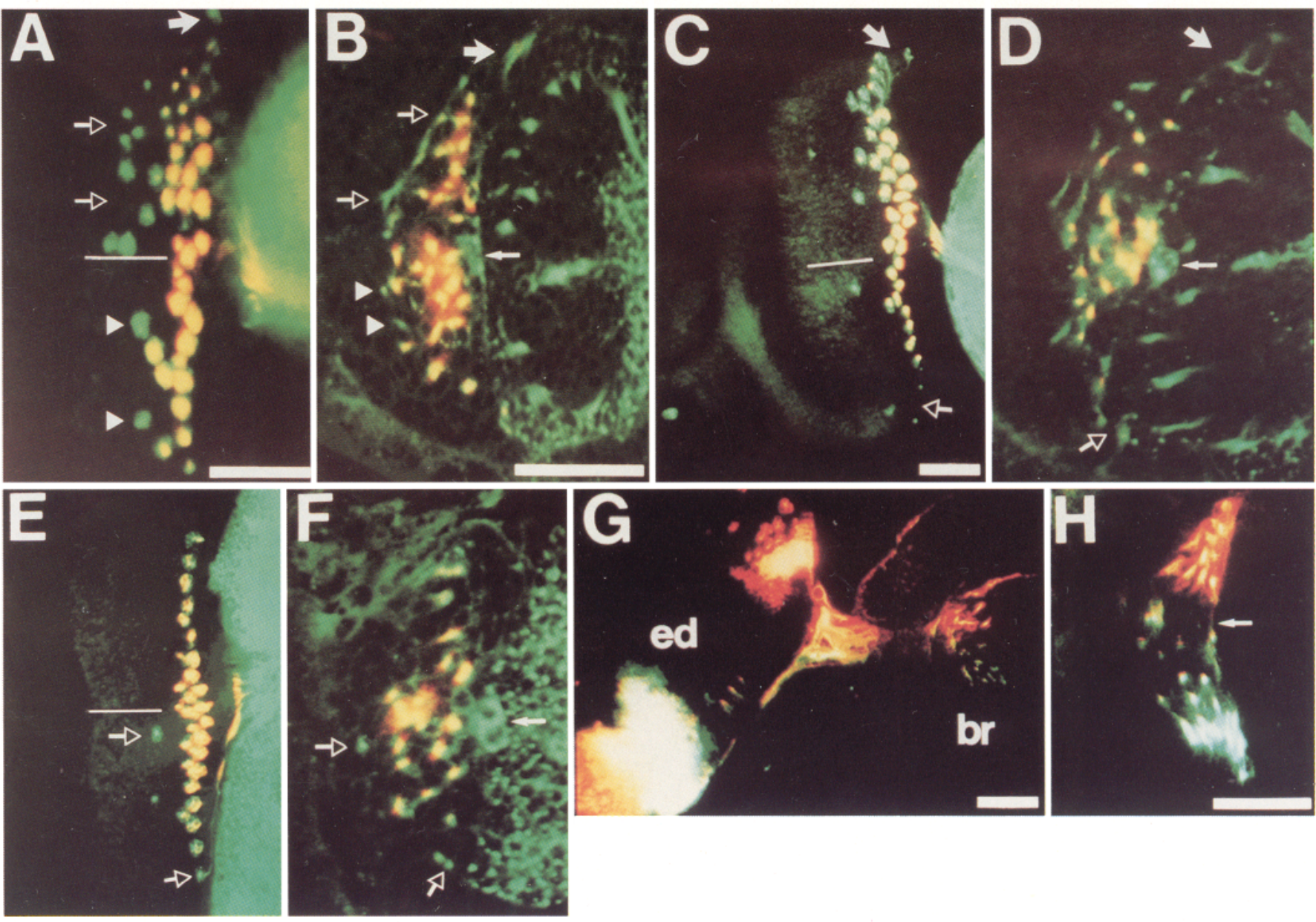
project posteriorly in close proximity to the OLPs, while younger fascicles are located in more anterior, dorsal, and ventral positions. These younger fascicles, in particular, are more widely spaced than in a wild-type brain. However, in some cases multifascicle retinal axon bundles from smaller clusters of anterior ommatidia do not separate normally in the brain, a phenomenon also observed in so.

In parallel with our experiments with so larvae, we have concentrated our studies on the dorsoventral projection pattern of retinal axons in Elp ${ }^{B I}$ larvae. In a preliminary analysis, two separate clusters of ommatidia within $E l p^{B 1}$ eye imaginal disks were labeled with the lipophilic dyes $\mathrm{DiI}$ and $\mathrm{DiO}$ (e.g., Fig. $9 G)$. In eight specimens analyzed by this approach, axons from dorsal ommatidia projected only dorsally, while axons from the ventral part of the eye disk projected ventrally (Fig. $9 H$ ). In a few cases, some of the more anterior labeled ommatidia appeared to be completely isolated from other ommatidia; however, it is not certain that all adjacent ommatidia are labeled by this technique. Nevertheless, our observations suggest that axons from most ommatidia in $E l p^{B 1}$ eye disks project retinotopically, even though many of these ommatidia are inappropriately separated from their neighbors during their early development.

Evidence for retinotopic projection of axons from isolated ommatidia in homozygous $E l p^{B I}$ larvae is also provided by analysis of whole-mount preparations stained with anti-HRP and $\mathrm{mAb} 24 \mathrm{~B} 10$. This is best illustrated by the small proportion of Elp eye disks (less than $5 \%$ of the 400 specimens examined) where the positioning of ommatidia along the dorsoventral axis is highly asymmetric. For example, in Figure $9 B$ the fascicles from most of the young ommatidis (stained only with anti-HRP) project in a broad array of anterodorsal locations, consistent with the concentration of these ommatidia in the dorsal half of the eye imaginal disk (Fig. 9A). Typically in Elp, many of the least mature ommatidia are found close to the dorsal and ventral edges of the eye disk (Fig. 9A,C). Remarkably, fascicles from these ommatidia frequently appear to project to extreme dorsal and ventral locations in the developing lamina, bypassing more centrally located unoccupied territory along their paths (see Fig. $9 B, D)$. This is particularly obvious for the ventral fascicles in Figure $9 D$, since relatively few ommatidia are positioned ventrally in the highly asymmetric eye disk.

In general, the staining patterns of photoreceptor clusters in Elp eye disks are too complex to identify an individual ommatidium and its fascicle unequivocally. It is usually only the asymmetric distribution of groups of ommatidia in some eye disks that allows us to conclude that axons project to their proper locations in the brain. However, Figure $9 E$ shows a rare example in which a central anterior ommatidium is one of only two ommatidia that are stained only with the anti-HRP antibody. In the brain, the positions of the two fascicles that do not stain with $\mathrm{mAb} 24 \mathrm{R} 10$ correspond well with these ommatidial locations. These data indicate that even isolated photoreceptor clusters with no near neighbors project retinotopically to the lamina in $E l p^{B \prime}$ larvae.

\section{Axonal projections in animals mosaic for the mutation glass}

Another approach to examining the role of axon-axon interactions in retinal axon guidance is to ask whether wild-type retinal axons project to their normal target positions when adjacent to mutant retinal axons that project aberrantly. To address this question, we examined animals that were mosaic for a mutation, $g l$, that results in retinal axon projection defects (Fig. 10B,C; Selleck and Steller, 1991). In addition, since the lack of $g l^{+}$function results in the absence of the larval optic nerve (Moses et al., 1989; A. R. Campos, personal communication), we could use $g l$ mosaics to assess the role of the larval optic nerve as a pioneer for retinal axon navigation in the brain.

In animals lacking the function of the $g l^{+}$gene, the pattern of retinal axon projections in the larval brain is extremely dis-

\footnotetext{
Figure 8. Retinal axon projections from small patches of developing ommatidia in so larvae. In the specimen shown in $A-C$, there are two separate patches of developing ommatidia on opposite sides of the dorsoventral midline (as defined by the position of the larval optic nerve, lon). $A$, In the eye disk the ventral patch, labeled with both $\mathrm{mAb} 24 \mathrm{~B} 10$ and anti-HRP (yellow), is slightly posterior to the dorsal patch (labeled with anti-HRP only). $B$, The axon fascicles from the respective patches traverse the optic stalk on opposite sides of the larval optic nerve and take opposing tracts in the brain toward appropriate dorsal (solid arrowhead) and ventral (open arrowhead) areas of the developing lamina. An arrow indicates the midline position of the OLP cells. $C$, A slightly deeper focal plane than in $B$, showing the dorsal $(d)$ and ventral $(v)$ fascicles as they pass through the lamina. $D$, The specimen shown in $D-F$ harbors a single patch of seven developing ommatidia (bracketed by open arrowheads) on the dorsal side of the larval optic nerve (lon). E, A montage of two focal planes showing the retinal fascicles as thcy traverse the optic stalk (os) alongside of the larval optic nerve and turn toward dorsal target sites (curved arrow) in the developing lamina. $F$, In a slightly deeper focal plane than that shown in $E$, the retinal fascicles are seen as they pass through the developing lamina (open arrowhead) in the dorsal region. The solid arrow marks the position of the dorsoventral midline. All panels are from a lateral aspect. Anterior is toward the left, dorsal is up. Scale bar, $25 \mu \mathrm{m}$.

Figure 9. Retinal projections from isolated ommatidia in the mutant $E l p . A, C, E$, and $G$ show confocal micrographs of eye imaginal disks from $E l p^{\mathrm{BI}}$ homozygous larvae. $B, D, F$, and $H$, respectively, are optical sections through the developing laminas of the corresponding brain hemispheres in a plane perpendicular to the paths of incoming retinal fascicles. For $A-F$, specimens were stained with mAb $24 \mathrm{~B} 10$ (red) and anti-HRP (green) antibodies. They provide several examples in which widely separated ommatidia in the eye disk appear to project to appropriate positions in the larval brain. In $A$ and $B$, a dorsal group of less mature widely spaced ommatidia (mAb $24 \mathrm{~B} 10$ negative) in $A$ and corresponding dorsally located fascicles in $B$ are marked by open arrows. Two ventral mAb 24B10-negative ommatidia and their likely projections in the brain are shown by arrowheads. In $D$, an open arrow indicates the position of fascicles that project to ventral locations, even though a ventral region of the target area lying closer to the midline is largely unoccupied by photoreceptor axons. The group of ommatidia from which these fascicles most likely grew is marked in $C$ by an open arrow. The cell bodies of some of these R-cells lie outside of the focal plane. The solid arrows in $A$ and $C$ indicate the positions of ommatidia whose fascicles target extreme dorsal locations (solid, thick arrows in $B$ and $D$ ). The two ommatidia in $E$ and corresponding fascicles in $F$ that are stained only by anti-HRP are marked by open arrows. In $G$, retinal axons in $E l p$ larvae were traced using the lipophilic dyes Dil and DiO, as described in Materials and Methods. In the eye disk (ed), a dorsal region was labeled with DiI (red to yellow fluorescence) and a ventral region was labeled with $\mathrm{DiO}$ (green fluorescence). The retinal fascicles project appropriately along dorsal (Dil) and ventral (DiO) paths through the optic stalk (mostly out of the focal plane) to dorsal and ventral regions of the developing lamina in the brain (br). $H$ is a highermagnification view of the developing lamina of the specimen shown in $G$. Fascicles from dorsal (DiI-labeled) ommatidia extend to dorsal target areas and ventral (DiO-labeled) fascicles extend to ventral targets areas. In $A, C$, and $E$, a thin bar marks the position of the larval optic nerve in the eye disk. In $B, D, F$, and $H$, the OLP cells are indicated by a thin, solid arrow. In all panels, anterior is to the left, dorsal is up. Scale bars: $A$, $C, G$ and $H, 25 \mu \mathrm{m} ; B, 20 \mu \mathrm{m}$ for $B, D$, and $F$.
} 

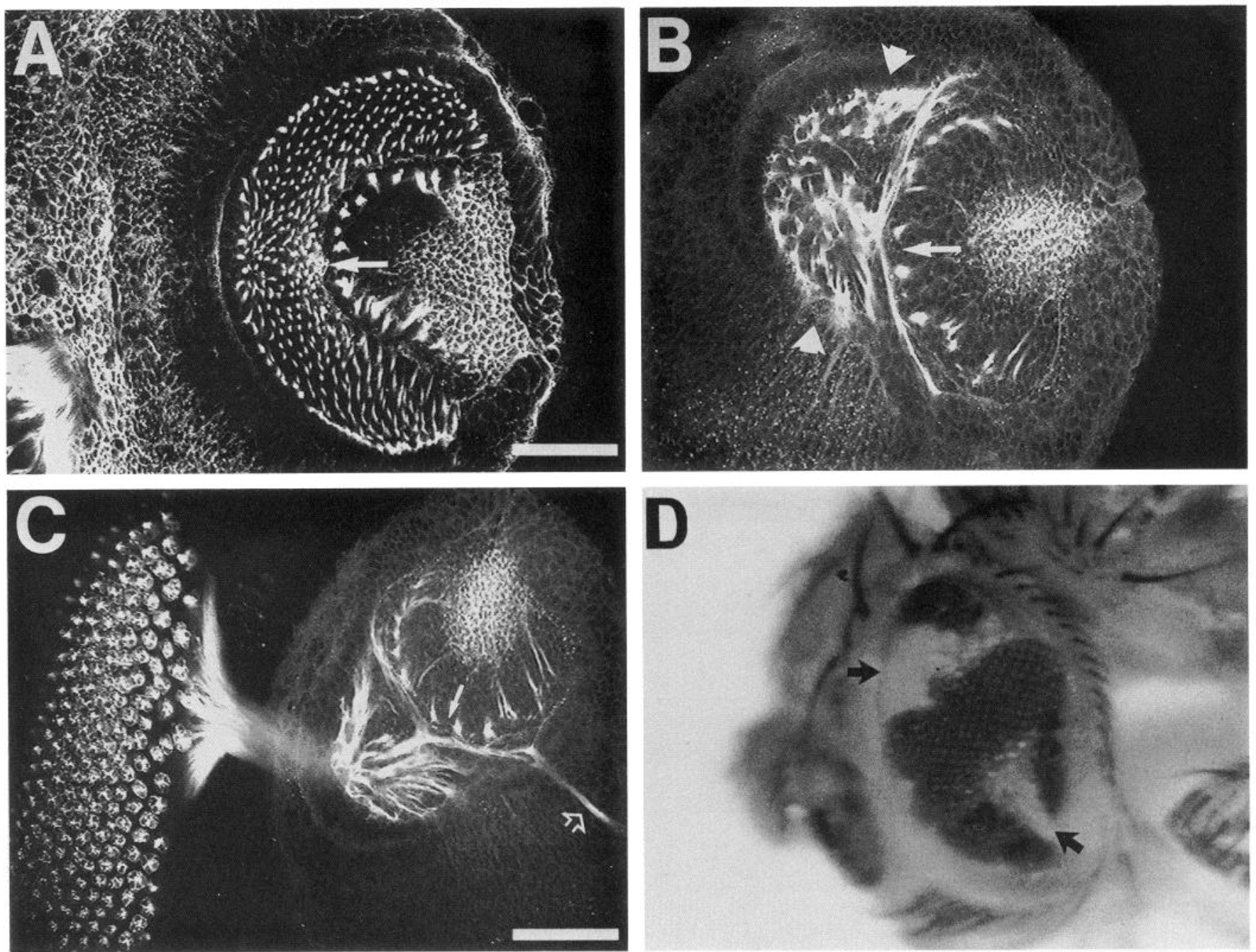

Figure 10. Retinal axon projections in a $g l$ mutant. $A$, Optical section through the developing lamina of a wild-type brain stained with anti-HRP. Arrow indicates the position of OLP cells posterior of the developing lamina at the dorsoventral midline (see Fig. $2 G$ for further details). $B$, The brain of a $g l^{60 J}$ animal in an optical section similar to that shown in $A$. Retinal axons form multifascicle bundles that take aberrant trajectories (area between arrowheads) through the developing lamina and often project ectopically out of the target area to other regions of the brain. $C$, A lower-magnification view of the specimen shown in $B$. The larval optic nerve is absent, and the OLPs cannot be detected. However, often in $g l^{603}$ specimens the OLPs are present in a normal or ectopic location. The open arrowhead indicates an ectopic retinal axon projection. $D$, Eye of an adult $g l$ mosaic generated as described in Materials and Methods. Regions of dark pigmented retinal tissue are $g l^{+}$, while $g l^{60 j}$ regions $(a r r o w s)$ lack pigmentation and are devoid of ommatidia. $g l$ cells form ommatidial clusters initially (as shown in $C$ ) but die during later development. All panels are from a lateral perspective. Anterior is toward the left, dorsal is up. In $C$ the brain is rotated slightly counterclockwise. Scale bars: $A$ and $B$, 25 $\mu \mathrm{m} ; C, 50 \mu \mathrm{m}$.

organized (compare Fig. 10B, $C$ with Fig. 10A). Axon fascicles take aberrant trajectories after they exit the optic stalk, and terminate in ectopic locations often outside of the lamina target area. The $g l$ locus is well characterized (Moses et al., 1989; Moses and Rubin, 1991). It encodes an apparent transcription factor required in R-cells for the expression of at least several R-cellspecific genes including chaoptic (Moses et al., 1989), which encodes the antigen recognized by $\mathrm{mAb} 24 \mathrm{~B} 10$ (Zipursky et al., 1985; Reinke et al., 1988). $g l$ is expressed in R-cells, in the larval optic nerve, and in a small subset of cells in the third instar larval brain (Moses and Rubin, 1991). Finally, the optic lobe and eye defects of adult $g l$ animals have been shown by the analysis of adult genetic mosaics to be due to the lack of $\mathrm{gl}^{+}$ function in the eye (Meyerowitz and Kankel, 1978).

To determine whether wild-type retinal axons project to their normal lamina target positions independently of the aberrant projections of $\mathrm{gl}^{-}$axons, we examined the developing brains of $\mathrm{gl}$ somatic mosaics. Mosaics were generated by inducing the somatic loss of a chromosomally inserted P-element vector harboring a $\mathrm{gl}^{+}$genomic DNA fragment (Moses et al., 1989; see Fig. 10D). P-transposase-mediated excision and loss of the $\mathrm{gl}^{+}$ element uncovers a $g l^{60 J}$ mutant background. Since $g l^{+}$function is required for chaoptin expression, a patch of $g l^{60 J} \mathrm{R}$-cells in the posterior of the eye disk can be recognized by its failure to stain with $\mathrm{mAb} 24 \mathrm{~B} 10$. Both wild-type and $g l^{600}$ retinal axons can be visualized by staining with anti-HRP.

In all of the mosaic animals examined, axonal projection defects were found to be closely associated with the absence of $\mathrm{gl}^{+}$function in R-cells. Of 850 eye imaginal disks, 775 harbored one or more $g l^{-}$patches. In 20 eye disks that appeared entirely $\mathrm{gl}^{+}$, retinal axon projections appeared normal (data not shown). Conversely, in the small number of cases (about 10) in which 
nearly all R-cells were $g l^{-}$(which can be determined only for those R-cells mature enough to express chaoptin), the retinal axon projections were as disorganized as in the $g l^{60 \mathrm{~J}}$ mutant (data not shown). However, these mosaics differ from $\mathrm{gl}^{\mathrm{loJ}^{\circ \mathrm{J}}}$ animals by the presence of the larval optic nerve. In five mosaics, the larval optic nerve could not be detected. In each of these animals, $g l^{+}$retinal axons formed essentially normal projections (e.g, Fig. $11 G, H$ ). Thus, the absence of the larval optic nerve in the third larval instar is not alone sufficient to cause the aberrant retinal axon projections found in $g l^{-}$animals. These last observations indicate that the larval optic nerve is not required as a pioneer in retinal axon guidance.

To assess the effect of $\mathrm{gl}^{-}$axons on neighboring wild-type axons, we examined genetic mosaics where rather large regions of $g l^{-} \mathrm{R}$-cells bordered regions of wild-type R-cells in the eye disk (Fig. $11 A-F$ ). This class includes about one-third of all those examined. In the specimen shown in Figure $11, A$ and $B$, the dorsal half of the eye disk is $\mathrm{gl}^{-}$except for a few $\mathrm{gl}^{+}$ommatidia (see inset, Fig. 11A). In the brain (Fig. 11B), the axons from the $\mathrm{gl}^{+}$ventral half of the eye disk form a normal array in the ventral portion of the developing lamina. The several isolated $g^{l+}$ fascicles from the dorsal portion of the eye disk project to appropriate dorsal positions despite the absence of most of their usual neighbors, which have failed to enter the dorsal target area. Notably, these $g l^{+}$axons did not follow $g l^{-}$ fascicles along their aberrant paths. Similar cases are shown in Figure $11 C-F$. In the specimen shown in Figure $11, E$ and $F$, $g l^{+}$retinal axons from ventral positions in the eye disk project to appropriate dorsoventral positions despite the projections of more posterior $g l^{-}$fascicles to aberrant positions. Hence, the more anterior $\mathrm{gl}^{+}$axons do not appear to rely on these posterior fascicles as pioneers. In the total of 250 mosaics in this class, wild-type retinal fascicles were found in approximately correct dorsoventral positions despite the aberrant behavior of their $\mathrm{gl}^{-}$ neighbors. In summary, our observations from the analysis of $g l$ mosaics support the proposal that axon fascicles are guided independently of their neighbors.

\section{Discussion}

We have sought to assess the importance of two different possible mechanisms for retinal axon guidance in Drosophila. One model proposes that interactions between retinal axons play the dominant role in guiding retinal axons to their target destinations (see Meinertzhagen, 1974; Anderson, 1978). Given the remarkably specific spatial and temporal order of retinal axon ingrowth to the developing optic lobe (Meinertzhagen, 1973; Trujillo-Cenóz and Melamed, 1973; Tomlinson and Ready, 1987), it might be supposed that newly arriving retinal axons are guided into place by the axon fascicles that have immediately preceded them into the brain. For example, the growth cones of a new fascicle could follow along the fascicle of a nearby morc mature ommatidium. On account of the one-to-one topographical order of the retinotopic map, the new fascicle would be led to a target position immediately adjacent to its proper destination. The guidance of the very first retinal fascicles might depend on their close association with the larval optic nerve, which passes near their target destinations at the posterior of the developing lamina near the dorsoventral midline (see Fig. 1A; Meinertzhagen, 1973, 1974). A second possible model of retinal axon guidance supposes that retinal fascicles seek out their respective target positions independently of one another, relying on positional cues that label the appropriate paths and target sites.

In an attempt to distinguish between these two possible guidance mechanisms, we used genetic methods to disrupt the normal spatial order of retinal axon outgrowth. We examined retinal axon projections within the developing lamina in two mutants in which reduced numbers of ommatidia develop in the eye imaginal disk. In so (sine oculis) animals, ommatidia develop in variously sized patches at different positions within the eye disk. In Elp (Ellipse) animals, single ommatidia develop at isolated locations in more anterior regions of the disk. In the lamina target area of both mutants, retinal axons were found in dorsoventral positions roughly corresponding to the positions of the remaining ommatidia in the eye disk. Due to the absence of reliable anteroposterior landmarks in the target area, the positions of the projections along this axis remain unclear, though the axons were ordered in their normal age-related pattern.

If the axons from the ommatidia remaining in these mutants were to navigate solely on the basis of the order of axon outgrowth and axon-axon interactions, we might have expected them to occupy the more central target positions left vacant by the absence of the corresponding ommatidia. Moreover, we would have expected the fascicles from isolated ommatidia to project to adjacent target positions rather than to spread out to more appropriate isolated positions. We thus suppose that, as they enter the target area, individual fascicles can make dorsoventral choices independently and that they bypass inappropriate vacant target sites as they grow along the lateral surface of the brain toward their proper destinations.

A concern with this interpretation is that the so and $E l p$ mutations might have effects on axon guidance via a functional role in the brain. In the case of $s o$, this would seem highly unlikely since genetic mosaic analysis has assigned the role of $\mathrm{SO}^{+}$in visual system development to the gene's function in the eye disk with no detectable gene autonomous function in brain development (Fischbach and Technau, 1984). By contrast, the observation that the $f l b^{+}$gene is expressed in the larval brain (Kammermeyer and Wadsworth, 1987; Katzen et al., 1991; Zak and Shilo, 1992) raises the possibility that the Elp mutation affects cells in the lamina target area. Nonetheless, it seems unlikely that the apparent independent guidance of retinal axons in Elp larvae does not reflect a similar process occurring in wildtype animals.

These conclusions are supported by the analysis of retinal axon guidance in $g l$ genetic mosaic animals. In larvae lacking the function of the $g l$ gene, retinal axons navigate aberrantly in the brain (Selleck and Steller, 1991) and the larval optic nerve is absent (Moses et al., 1989). In mosaics, we observed that genotypically wild-type retinal axons can project normally when adjacent to misrouted $g l^{-}$axons, consistent with the hypothesis that retinal fascicles can navigate independently of one another. These observations arc in agrecment with the conclusions of Meyerowitz and Kankel (1978) from the analysis of adult $g l$ mosaic animals. In their study, areas of aberrant lamina neuropil were found to correspond retinotopically to $\mathrm{gl}$ - regions of the retina. Though their experiments involved a hypomorphic $g l$ allele, $g l^{3}$, that displays rather minor defects in the retinal axon projection pattern of late third instar larvae (S. Kunes, C. Wilson, and H. Steller, unpublished observations), the outcome nonetheless is consistent with the proposal that $\mathrm{gl}^{+}$functions autonomously in photoreceptor cells for retinal axon guidance. Since the $\mathrm{gl}^{+}$gene product appears to be a transcription factor 

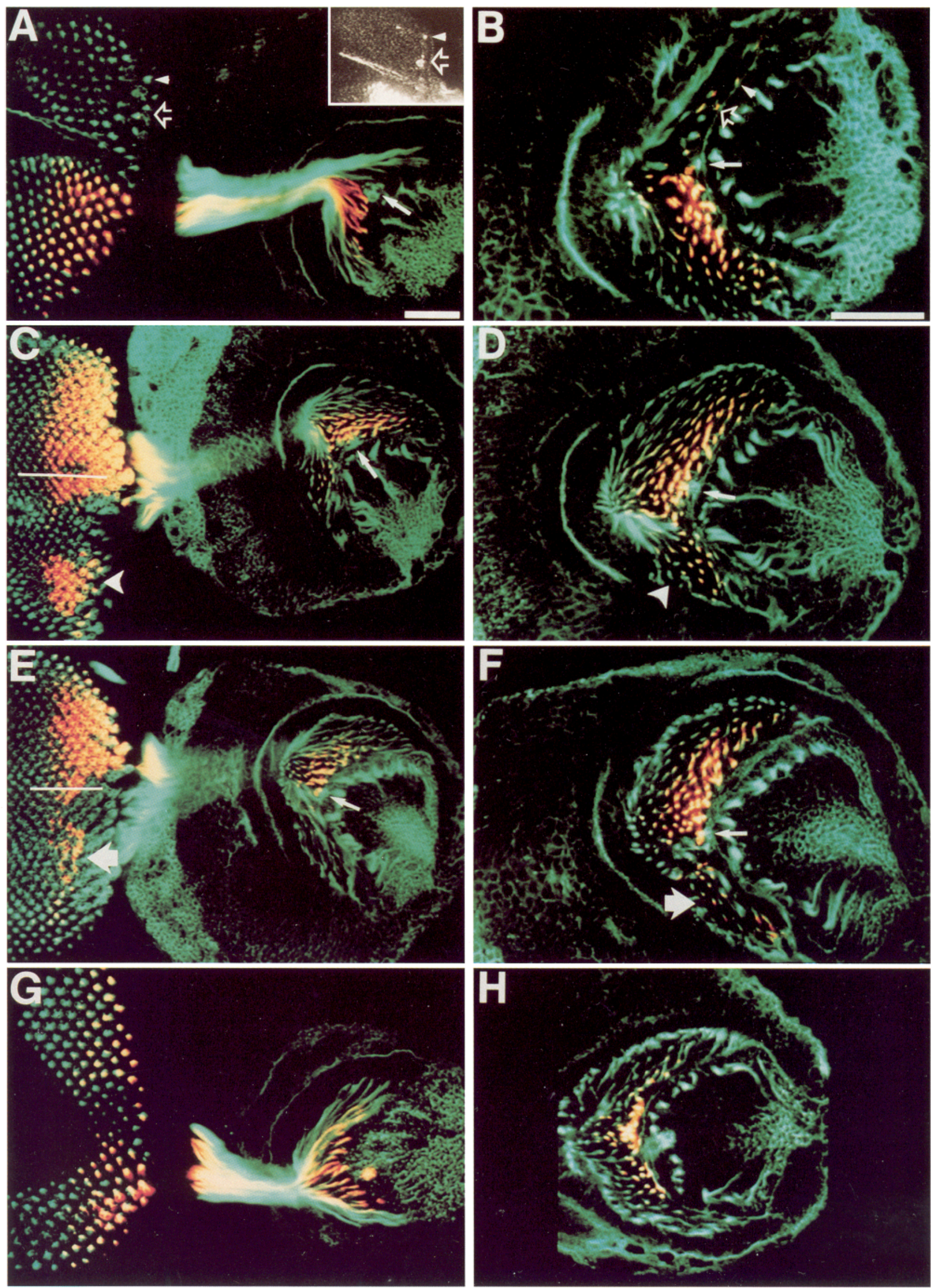
involved in the regulation of photoreceptor cell-specific genes (Moses et al., 1989; Moses and Rubin, 1991), it seems likely that $g l^{-}$photoreceptor cells fail to express an as yet unidentified gene product(s) that is essential for axon pathfinding.

In addition, the analysis of $g l$ mosaic animals lends support to the notion that the larval optic nerve is not an essential pioneer for retinal axon navigation. Wild-type retinal fascicles are found in approximately normal dorsoventral locations in animals lacking the larval optic nerve due to its inclusion in $\mathrm{gl}^{-}$ tissue. It was initially evident from the analysis of $g l^{-}$animals that the navigation of retinal axons through the optic stalk did not require the presence of the larval optic nerve (Moses et al., 1989). From the mosaic analysis, it can now be concluded that the larval optic nerve is not essential for retinal axons to establish their normal projection pattern when they reach the optic lobe. A normal retinal axon projection pattern is also observed after the larval optic nerve has been ablated by the expression of a toxin gene (Kunes and Steller, 1991). The lack of a requirement for the larval optic nerve is not surprising in view of other examples in which later-arriving axons traverse their usual paths in the absence of earlier axons from the same pathway (Keshishian and Bentley, 1983; Blair et al., 1987; Tix et al., 1989b; Pike et al., 1992).

It has been proposed that the spatial and temporal order of retinal axon ingrowth observed in several arthropod visual systems might alone be sufficient to explain the initial order of their respective retinal axon projection patterns (Meinertzhagen, 1973, 1974; Anderson, 1978; Macagno, 1978; Flaster et al., 1982). Indeed, several experiments appear to bear out the predictions of this hypothesis. In the small crustacean, Daphnia, later-arriving photoreceptor axons will assume the target cell contacts normally reserved for earlier axons if the latter have been ablated earlier in development (Macagno, 1978). In the locust Schistocerca, transplantation and ablation experiments can be interpreted as supporting the idea that new retinal axons are guided by following the projections of older ommatidia, rather than seeking out their lamina target sites independently (Anderson, 1978). Although our results argue against an essential role for retinal axon-axon interactions in Drosophila, they may not be entirely inconsistent with the previous experiments in arthropods. For example, we have not resolved the question of target finding along the anteroposterior axis of the lamina in Drosophila, which would be directly comparable to the study in Daphnia (Macagno, 1978). In addition, our study does not pro- vide the resolution necessary to determine whether fascicles seek out specific target cells in either the wild-type or mutant animals. In Schistocerca, axons derived from retinal transplants rotated on the dorsoventral axis apparently failed to find their normal lamina targets. However, in these experiments the target area was always damaged to some extent and the projections from the rotated epithelia were often highly abnormal (Anderson, 1978). This kind of experiment, moreover, provides a rather stringent test of the independence of retinal axon navigation since proper target finding would require the axons to seek out their appropriate target positions via ectopic pathways. Such was not the case in the experiments reported here. It would thus be difficult to conclude that the outcome of the transplantation experiments are inconsistent with our results. Evidence consistent with the independent guidance of retinal axons in Drosophila comes from the study of adult animals mosaic for either the Glued or rough mutations, where areas of aberrant lamina neuropil were found to correspond retinotopically to mutant regions of the retina (Meyerowitz and Kankel, 1978; Harte and Kankel, 1983).

Given these considerations, it is reasonable to suppose that multiple mechanisms may contribute to retinal axon guidance in Drosophila. It seems unlikely that the normal morphogenetic order of retinal axon ingrowth is merely incidental to proper target finding. In addition, our observations are consistent with a contribution of fascicle-fascicle interactions to the formation of precise retinotopic projections. In $s o$ animals harboring very few developing ommatidia and in some Elp animals, retinal fascicles failed to separate normally into single fascicles after entering the developing lamina. Normal specificity may rely on fascicle-fascicle interactions that occur in arrays containing a larger number of adjacent fibers. A retinal growth cone may assess the position(s) of a neighboring fascicle(s) as well as guidance cues that label pathways and target sites in the brain. The latter kind of mechanism could involve a large number of unique guidance markers (sce Spcrry, 1963, for a discussion) or a small number of molecular components if they are distributed in a graded fashion in the target area (Sperry, 1963; Gierer, 1981; Bonheoffer and Gierer, 1984; Gierer, 1987). As has been suggested for lower vertebrates (see, e.g., Fraser, 1985), a mechanism involving both interactions between retinal axon fibers and position-specific fiber-target interactions may ultimately provide the most parsimonious explanation of retinal axon guidance in Drosophila.

\footnotetext{
Figure 11. Retinal axon projections in $g l$ genetic mosaics. $g l$ genetic mosaics were generated as described in Materials and Methods. Late third instar larval tissues were labeled with $\mathrm{mAb} 24 \mathrm{~B} 10$ (red fluorescence) and anti-HRP (green fluorescence). Labeled specimens were viewed by confocal laser microscopy from thc latcral pcrspcctivc. Except in the anterior six or seven rows of developing ommatidia, $g l^{+}$photoreceptor cells express the antigens recognized by both $\mathrm{mAb} 24 \mathrm{~B} 10$ and anti-HRP, and thus are double labeled (yellow). $A, g l$ mosaic in which most ommatidia in the dorsal part of the eye disk are $g l^{600}$. However, four dorsal ommatidia (open and solid arrowheads; see also inset showing mAb 24B10 fluorescence) appear $g l^{+}$. Note that ventral $g l^{+}$fascicles project along the ventral side of the optic stalk to ventral regions of the developing lamina. The larval optic nerve is present. $B$, Higher-magnification view of an optical section through the developing lamina of the specimen shown in $A$. Ventral $g l^{+}$ fascicles project to normal positions. Despite the aberrant projections of most $g l^{-}$dorsal fascicles (not detected in this focal plane), the four $g l^{+}$ dorsal fascicles project to appropriate positions (open and solid arrowheads). $C, g l$ mosaic in which a ventral area of $g l^{+}$developing ommatidia (arrowhead) is surrounded on both sides by regions of $\mathrm{gl}^{-}$developing ommatidia. Thin bar indicates the position of the larval optic nerve (out of the focal plane). $D$. Higher-magnification view of the developing lamina of the specimen shown in $C$. Despite the aberrant projections of the ventral $g l^{-}$fascicles, the ventral (arrowhead) and dorsal $g l^{+}$fascicles project normally. $E$, A case similar to that described in $C$ and $D$, except that the posterior four to five rows of ventral ommatidia are $\mathrm{gl}^{-}$(thick arrow indicates $g \mathrm{l}^{+}$ommatidia). $F$, Higher-magnification view of the developing lamina of the specimen shown in $E$. Despite the aberrant projections of posterior $g l^{-}$ventral fascicles, the ventral $\mathrm{gl} l^{+}$fascicles form an essentially normal dorsoventral array more anteriorly (thick arrow). $G, g l$ mosaic in which the larval optic nerve is absent. The eye disk also harbors a central patch of $g l^{-}$ommatidia. $H$, Higher-magnification view of the developing lamina of the specimen shown in $G$. $g l^{+}$retinal fascicles project in an approximatcly normal array despite the absence of the larval optic nerve. The OLP cells are absent. In all panels, anterior is toward the left, dorsal is up. In $A-F$, a thin arrow indicates the position of the OLP cells. Scale bars: $A, 25 \mu \mathrm{m}$ for $A, C, E$, and $G ; B, 25 \mu \mathrm{m}$ for $B, D, F$, and $H$.
} 
Our observations reveal features of retinotopic pattern formation in the Drosophila visual system that are reminiscent of the retinotectal system long studied in lower vertebrates. The experiments in Drosophila and in lower vertebrates (reviewed in Cowan and Hunt, 1985; Udin and Fawcett, 1988) both suggest that positional information in the retina and in the brain contribute to the establishment of well-ordered retinotopic connections. Whether these parallels reflect the operations of a similar molecular machinery would be an interesting question to resolve.

\section{References}

Anderson H (1978) Postembryonic development of the visual system of the locust, Schistocerca gregaria. II. An experimental investigation of the formation of the retina-lamina projection. J Fmbryol Fxp Morphol 46:147-170.

Ashburner M (1989) Drosophila: a laboratory manual. Cold Spring Harbor, NY: Cold Spring Harbor Laboratory.

Attardi DG, Sperry RW (1963) Preferential selection of central pathways by regenerating optic fibers. Exp Neurol 7:46-64.

Baker NE, Rubin GM (1989) Effect on eye development of dominant mutations in Drosophila homologue of the EGF receptor. Nature 340: 150-153.

Blair SS, Murray MA, Palka J (1987) The guidance of axons from transplanted neurons through aneural Drosophila wings. J Neurosci $7: 4165-4175$.

Bodick N, Levinthal C (1980) Growing optic nerve fibers follow neighbors during embryogenesis. Proc Natl Acad Sci USA 77:4374-4378.

Bolwig N (1946) Senses and sense organs of the anterior end of the housefly larvae. Vidensk Medd Dan Naturhist Foren 109:81-217.

Bonhoeffer F, Gierrer A (1984) How do retinal axons find their targets on the tectum? Trends Neurosci 7:378-381.

Bonhoeffer $\mathrm{F}$, Huf J (1982) In vitro experiments on axon guidance and tectal gradients demonstrating an anterior posterior gradient on the tectum. EMBO J 1:427-431.

Braitenberg V (1967) Patterns of projections in the visual system of the fly. 1. Retina-lamina projections. Exp Brain Res 3:271-298.

Bunt SM, Horder TJ, Martin KAC (1978) Evidence that optic fibers regenerating across the goldfish tectum may be assigned termination sites on a 'first come, first served' basis. J Physiol (Lond) 276:45P$46 \mathrm{P}$.

Cline TW (1978) Two closely-linked mutations in Drosophila melanogaster that are lethal to opposite sexes and interact with daughterless. Genetics 90:683-698.

Cowan WM, Hunt RK (1985) The development of the retinotectal projection: an overview. In: Molecular bases of neural development (Edelman GM, Gall WE, Cowan WM, eds), pp 389-428. New York: Wiley.

Fischbach K-F, Technau G (1984) Cell degeneration in the developing optic lobes of the sine oculis and small-optic-lobes mutants of Drosophila melanogaster. Dev Biol 104:219-239.

Fischbach KF, Dittrich APM (1989) The optic lobe of Drosophila metanogaster. I. A Golgi analysis of wild-type structure. Cell Tissue Res 258:441-475.

Flaster MS, Macagno ER, Schehr RS (1982) Mechanisms for the formation of synaptic connections in the isogenic nervous system of Daphnia magna. In: Neuronal development (Spitzer NC, ed), pp $267-$ 296. New York: Plenum.

Fraser SE (1985) Cell interactions involved in neuronal patterning: an experimental and theoretical approach. In: Molecular bases of neural development (Edelman GM, Gall WE, Cowan WM, eds), pp 481507. New York: Wiley.

Fujita SC, Zipursky SL, Benzer S, Ferrus A, Shotwell S (1982) Monoclonal antibodies against the Drosophila nervous system. Proc Natl Acad Sci USA 79:7929-7933.

Gaze RM (1960) Regeneration of the optic nerve in amphibia. Int Rev Neurobiol 2:1-40.

Gierer A (1981) Development of projections between areas of the nervous system. Biol Cybern 42:69-78.

Gierer A (1987) Direction cues for growing axons forming the retinotectal projection. Development 101:479-489.
Godement P, Vanselow J, Thanos S, Bonhoeffer F (1987) A study in developing visual systems with a new method of staining neurones and their processes in fixed tissue. Development 101:697-713.

Goodman CS, Bastiani MJ, Doe CQ, Du Lac S, Helfand SL, Kuwada JY, Thomas JB (1984) Cell recognition during neuronal development. Science 225:1271-1279.

Grant P, Rubin E (1980) Ontogeny of the retina and optic nerve in Xenopus laevis. II. Ontogeny of the optic nerve fiber pattern in the retina. J Comp Neurol 189:671-698.

Grenningloh G, Rehm EJ, Goodman CS (1991) Genetic analysis of growth cone guidance in Drosophila: fasciclin II functions as a neuronal recognition molecule. Cell 67:45-57.

Harte PJ, Kankel DR (1983) Analysis of visual system development in Drosophila melanogaster. mutations at the Glued locus. Dev Biol 99:88-102.

Honig MG, Hume RI (1986) Fluorescent carbocyanine dyes allow living neurons of identified origin to be studied in long-term culture. J Cell Biol 103:171-187.

Horder TJ, Martin KAC (1978) Morphogenetics as an alternative to chemospecificity in the formation of nerve connections. Symp Soc Exp Biol 32:275-359.

Horridge GA, Meinertzhagen IA (1970) The accuracy of the patterns of connexions of the first- and second-order neurons of the visual system of Calliphora. Proc R Soc Lond [Biol] 175:69-82.

Jan LY, Jan YN (1982) Antibodies to horseradish peroxidase as specific neuronal markers in Drosophila and in grasshopper embryos. Proc Natl Acad Sci USA 79:2700-2704.

Johnson GD, Araujo GM (1981) A simple method of reducing the fading of immunofluorescence during microscopy. J Immunol Methods 43:349-350.

Kammermeyer KL, Wadsworth SC (1987) Expression of Drosophila epidermal growth factor receptor homologue in mitotic cell populations. Development 100:201-210.

Katzen AL, Kornberg T, Bishop JM (1991) Expression during Drosophila development of DER, a gene related to erbB-1 and neu: correlations with mutant phenotypes. Dev Biol 145:287-301.

Keshishian H, Bentley D (1983) Embryogenesis of peripheral nerve pathways in grasshopper legs. III. Development without pioneer neurons. Dev Biol 96:116-124.

Kunes S, Steller H (1991) Ablation of Drosophila photoreceptor cells by conditional expression of a toxin gene. Genes Dev 5:970-983.

Laski FA, Rio DC, Rubin GM (1986) Tissue specificity of Drosophila P-element transposition is regulated at the level of mRNA splicing. Cell 44:7-19.

Lindsley DL, Zimm GG (1992) The genome of Drosophila melanogaster, pp 645-655. San Diego: Academic.

Macagno ER (1978) Mechanism for the formation of synaptic projections in the arthropod visual system. Nature 275:318-320.

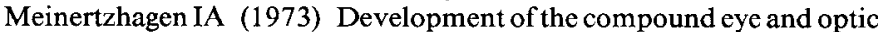
lobe of insects. In: Developmental neurobiology of arthropods (Young D, ed), pp 52-104. Cambridge: Cambridge UP.

Meinertzhagen IA (1974) The development of neuronal connection patterns in the visual systems of insects. In: CIBA Foundation symposium 29 (new series), Cell patterning, pp 265-288. New York: Elsevier.

Meyerowitz EM, Kankel D (1978) A genetic analysis of visual system development in Drosophila melanogaster. Dev Biol 62:112-142.

Moses K, Rubin GM (1991) glass encodes a site-specific DNA-binding protein that is regulated in response to positional signals in the developing Drosophila eye. Genes Dev 5:583-593.

Moses K, Ellis MC, Rubin GM (1989) The glass gene encodes a zincfinger protein required by Drosophila photoreceptor cells. Nature 340: $531-536$.

Pike SH, Melancon EF, Eisen JS (1992) Pathfinding by zebrafish motoneurons in the absence of normal pioneer axons. Development 114: $825-831$.

Price JV, Clifford RJ, Schupbach T (1989) The maternal ventralizing locus torpedo is allelic to faint little ball, an embryonic lethal, and encodes the Drosophila EGF receptor homolog. Cell 56:1085-1092.

Ramón y Cajal S, Sánchez y Sánchez D (1915) Contribucion al conocimiento de los centros nerviosos de los insectos. Trab Inst Cajal Invest Biol 13:1-164.

Ready DF (1989) A multifaceted approach to neural development. Trends Neurosci 12:102-110. 
Ready DF, Hanson TE, Benzer S (1976) Development of the Drosophila retina, a neurocrystalline lattice. Dev Biol 53:217-240.

Reinke R, Krantz DE, Yen D, Zipursky SL (1988) Chaoptin, a cell surface glycoprotein required for Drosophila photoreceptor cell morphogenesis, contains a repeat motif found in yeast and human. Cell 52:291-301.

Robertson HM, Preston CR, Phillis RW, Johnson-Schiltz DM, Benz WK, Engels WR (1988) A stable source of P-element transposase in Drosophila melanogaster. Genetics 118:461-470.

Rubin GM (1989) Development of the Drosophila retina: inductive events studied at single cell resolution. Cell 57:519-520.

Schejter ED, Shilo B-Z (1989) The Drosophila EGF receptor homolog (DER) gene is allelic to faint little ball, a locus essential for embryonic development. Cell 56:1093-1104.

Selleck SB, Steller H (1991) The influence of retinal innervation on neurogenesis in the first optic ganglion of Drosophila. Neuron 6:8399.

Sims PJ, Waggoner AS, Wang CH, Hoffman JF (1974) Studies on the mechanism by which cyanine dyes measure membrane potential in red blood cells and phosphatidylcholine vesicles. Biochemistry 13: $3315-3330$.

Sperry RW (1963) Chemoaffinity in the orderly growth of nerve fiber. patterns and connections. Proc Natl Acad Sci USA 50:703-710.

Stahl B, Muller B, von Boxberg Y, Cox EC, Bonheoffer F (1990) Biochemical characterization of a putative axonal guidance molecule of the chick visual system. Neuron 5:735-743.

Steller H, Fischbach K-F, Rubin GM (1987) disconnected, a locus required for neuronal pathway formation in the visual system of Drosophila. Cell 50:1139-1153.

Stirling RV (1991) Molecules, maps and gradients in the retinotectal projection. Trends Neurosci 14:509-512.
Strausfeld NJ (1976) Atlas of an insect brain. New York: Springer. Stuermer CAO, Easter SS Jr (1984) Rules of order in the retinotectal fascicles of goldfish. J Neurosci 4:1045-1051.

Tix S, Minden JS, Technau GM (1989a) Pre-existing neuronal pathways in the developing optic lobes of Drosophila. Development 105: $739-746$.

Tix S, Bate M, Technau GM (1989h) Pre-existing neuronal pathways in the leg imaginal discs of Drosophila. Development 107:855-862.

Tomlinson A (1988) Cellular interactions in the developing Drosophila eye. Development 104:183-193.

Tomlinson A, Ready DF (1987) Cell fate in the Drosophila ommatidium. Dev Biol 123:264-275.

Trujillo-Cenóz O, Melamed J (1966) Compound eye of dipterans: anatomical basis for integration - an electron microscope study. $\mathbf{J}$ Ultrastruct Res 16:395-398.

Trujillo-Cenóz O, Melamed J (1973) The development of the retinalamina complex in muscoid flies. J Ultrastruct Res 42:554-581.

Udin SB, Fawcett JW (1988) Formation of topographic maps. Annu Rev Neurosci 11:289-327.

Vielmetter J, Stuermer CAO (1989) Goldfish retinal axons respond to position-specific properties of tectal cell membranes in vitro. Neuron 2:1331-1339.

Zak NB, Shilo B-Z (1992) Localization of DER and the pattern of cell divisions in wild-type and Ellipse eye imaginal discs. Dev Biol 149: $448-456$.

Zipursky S, Venkatesh T, Teplow DB, Benzer S (1984) Neuronal development in the Drosophila retina: monoclonal antibodies as molecular probes. Cell 36:15-26.

Zipursky S, Venkatesh T, Benzer S (1985) From monoclonal antibody to gene for a neuron-specific glycoprotein in Drosophila. Proc Natl Acad Sci USA 82:1855-1859. 